Алгебра и анализ

Том. 16 (2004), вып. 1
St. Petersburg Math. J.

Vol. 16 (2005), No. 1, Pages 237-257

S 1061-0022(04)00849-0

Article electronically published on December 17, 2004

\title{
ON HOMOGENIZATION FOR A PERIODIC ELLIPTIC OPERATOR IN A STRIP
}

\author{
T. A. SUSLINA
}

Dedicated to my dear teacher Mikhail Shlemovich Birman with love and gratitude

\begin{abstract}
In a strip $\Pi=\mathbb{R} \times(0, a)$, the operator
$A_{\varepsilon}=D_{1} g_{1}\left(x_{1} / \varepsilon, x_{2}\right) D_{1}+D_{2} g_{2}\left(x_{1} / \varepsilon, x_{2}\right) D_{2}$

is considered, where $g_{1}, g_{2}$ are periodic with respect to the first variable. Periodic boundary conditions are put on the boundary of the strip. The behavior of the operator $A_{\varepsilon}$ in the limit $\varepsilon \rightarrow 0$ is studied. It is proved that, with respect to the operator norm in $L_{2}(\Pi)$, the resolvent $\left(A_{\varepsilon}+I\right)^{-1}$ tends to the resolvent of the effective operator $A^{0}$. A sharp order estimate for the norm of the difference of the resolvents is obtained. The operator $A^{0}$ is of the same type, but its coefficients depend only on $x_{2}$.
\end{abstract}

\section{$\S 0$. INTRODUCTION}

0.1. The problem of homogenization for periodic operators of mathematical physics is of significant interest both from the general-theoretic viewpoint and for applications. An extensive body of literature is devoted to homogenization problems. At the first place, the books [1-3] should be mentioned. A typical homogenization problem looks like this. Consider the family of operators

$$
\mathcal{A}_{\varepsilon}=-\operatorname{div} g(\mathbf{x} / \varepsilon) \operatorname{grad}, \quad \mathbf{x} \in \mathbb{R}^{d}, \varepsilon>0,
$$

where $g(\mathbf{x})$ is a positive definite bounded matrix-valued function periodic with respect to some lattice in $\mathbb{R}^{d}$. The problem is to find a constant positive matrix $g^{0}$ such that the resolvent $\left(\mathcal{A}_{\varepsilon}+I\right)^{-1}$ converges (in some sense) as $\varepsilon \rightarrow 0$ to the resolvent of the operator $\mathcal{A}^{0}=-\operatorname{div} g^{0}$ grad. The operator $\mathcal{A}^{0}$ is called the effective or homogenized operator.

A possible approach to homogenization of periodic differential operators in $\mathbb{R}^{d}$ consists in application of the Floquet theory. In this connection, we mention the papers [4] -8 . In [7, 8, it was proposed to treat the homogenization procedure as a threshold effect near the lower edge of the spectrum of a periodic differential operator. Namely, let $\Omega$ be the cell of the lattice of periods, and let $\mathcal{A}(\mathbf{k})$ be the family of operators acting in $L_{2}(\Omega)$ that appear in the direct integral decomposition for the operator $\mathcal{A}=-\operatorname{div} g(\mathbf{x}) \operatorname{grad}$. The parameter $\mathbf{k} \in \mathbb{R}^{d}$ is called the quasimomentum. The operator $\mathcal{A}(\mathbf{k})$ is defined by the expression $(\mathbf{D}+\mathbf{k})^{*} g(\mathbf{x})(\mathbf{D}+\mathbf{k})$ and periodic boundary conditions. The homogenization problem for $\mathcal{A}$ reduces to the study of a (two-parameter) family of operators $\left(\mathcal{A}(\mathbf{k})+\varepsilon^{2} I\right)^{-1}$ acting in $L_{2}(\Omega)$. Here, an important role is played by distinguishing the spectral germ of the family $\mathcal{A}(\mathbf{k})$ at $\mathbf{k}=0$. In terms of the spectral germ, we can find a good approximation for the resolvent $\left(\mathcal{A}(\mathbf{k})+\varepsilon^{2} I\right)^{-1}$. Since the effective operator $\mathcal{A}^{0}$ has the same spectral germ, this

2000 Mathematics Subject Classification. Primary 35B27.

Key words and phrases. Periodic operator, homogenization, effective operator.

Supported by RFBR (grant no. 02-01-00798). 
leads to approximation of the resolvent $\left(\mathcal{A}(\mathbf{k})+\varepsilon^{2} I\right)^{-1}$ by the resolvent $\left(\mathcal{A}^{0}(\mathbf{k})+\varepsilon^{2} I\right)^{-1}$ of the effective operator. As a result, for the initial operator (0.1) acting in $L_{2}\left(\mathbb{R}^{d}\right)$, the facts established in [7, 8] give convergence of the resolvent in the operator norm with the following sharp order estimate:

$$
\left\|\left(\mathcal{A}_{\varepsilon}+I\right)^{-1}-\left(\mathcal{A}^{0}+I\right)^{-1}\right\|_{L_{2}\left(\mathbb{R}^{d}\right) \rightarrow L_{2}\left(\mathbb{R}^{d}\right)} \leq C \varepsilon .
$$

0.2. Along with problems in the entire space $\mathbb{R}^{d}$, periodic problems can be considered in domains of cylinder type, layer type, etc., where the coefficients of the operator are periodic only in part of variables. In such problems, the effective operator depends on the "nonperiodic" variables, while homogenization is related to the "periodic" variables.

In the present paper, for a simplest model, we try to apply the approach of [7] [8] to such problems. We study the two-dimensional operator $A=-\operatorname{div} g\left(x_{1}, x_{2}\right) \operatorname{grad}$ in the strip $\Pi=\left\{\left(x_{1}, x_{2}\right): x_{1} \in \mathbb{R}, x_{2} \in(0, a)\right\}$. The matrix $g(\mathbf{x})$ is periodic along this strip (with respect to $x_{1}$ ) with period 1 . On the boundary $\partial \Pi$, we put periodic boundary conditions. The problem is to study the behavior of the operator

$$
A_{\varepsilon}=-\operatorname{div} g\left(x_{1} / \varepsilon, x_{2}\right) \operatorname{grad}
$$

as $\varepsilon \rightarrow 0$. It is assumed that the matrix $g(\mathbf{x})$ is diagonal: $g(\mathbf{x})=\operatorname{diag}\left\{g_{1}(\mathbf{x}), g_{2}(\mathbf{x})\right\}$. It turns out that the effective operator $A^{0}$ is of the form $A^{0}=-\operatorname{div} g^{0}\left(x_{2}\right) \operatorname{grad}$, where $g^{0}\left(x_{2}\right)=\operatorname{diag}\left\{g_{1}^{0}\left(x_{2}\right), g_{2}^{0}\left(x_{2}\right)\right\}$. Using the standard rules of homogenization theory, it is not difficult to find (formally) the expression for $g^{0}\left(x_{2}\right)$.

Our goal in the present paper is to obtain an estimate of sharp order for the difference of the resolvents of $A_{\varepsilon}$ and $A^{0}$ in the operator norm in $L_{2}(\Pi)$. The difficulties we encounter are related to the fact that the method of [7, 8, does not automatically yield the result.

In this paper, for the simple model problem just described, we show how the estimate

$$
\left\|\left(A_{\varepsilon}+I\right)^{-1}-\left(A^{0}+I\right)^{-1}\right\|_{L_{2}(\Pi) \rightarrow L_{2}(\Pi)} \leq C \varepsilon
$$

can still be obtained. We employ the results of [7, 8, but in combination with other methods. This requires some smoothness assumptions on the matrix-valued function $g(\mathbf{x})$ (see condition (1.4) below).

Now for the cell $\Omega$ we take $(0,1) \times(0, a)$, and the parameter $k$ is one-dimensional. After reducing the problem to the cell, we need to study a (two-parameter) family

$$
A(k, \varepsilon)=A_{1}(k)+\varepsilon^{2} A_{2}
$$

of operators acting in $L_{2}(\Omega)$. Here $A_{1}(k)=\left(D_{1}+k\right) g_{1}(\mathbf{x})\left(D_{1}+k\right)$ and $A_{2}=D_{2} g_{2}(\mathbf{x}) D_{2}$; the boundary conditions are periodic in both variables. The method of [7, 8 ] can be applied to the operator $A_{1}(k)$ "layerwise". This allows us to obtain a good approximation for $A_{1}(k)$ for small $|k|$ in terms of the corresponding spectral germ. The main technical difficulties are related to adding the unbounded "perturbation" $\varepsilon^{2} A_{2}$ and approximating the resolvent $\left(A(k, \varepsilon)+\varepsilon^{2} I\right)^{-1}$.

0.3. In $\S 1$, we state the problem and the main result. In $\S 2$, the problem is reduced to the study of the resolvent $\left(A(k, \varepsilon)+\varepsilon^{2} I\right)^{-1}$. In $\S 3$, the operator pencil $A_{1}(k)$ is treated in detail. In $\S 4$, approximation for the family $\left(A(k, \varepsilon)+\varepsilon^{2} I\right)^{-1}$ is constructed in terms of the spectral germ. $\S \S 5$ and 6 are devoted to auxiliary technical estimates. The proof of the main theorem is completed in $\S 7$. In $\S 8$, possible generalizations are discussed.

0.4. Notation. Let $\mathfrak{H}$ and $\mathfrak{G}$ be separable Hilbert spaces. The symbols $(\cdot, \cdot)_{\mathfrak{H}}$ and $\|\cdot\|_{\mathfrak{H}}$ stand for the inner product and the norm in $\mathfrak{H}$; the symbol $\|\cdot\|_{\mathfrak{H} \rightarrow \mathfrak{G}}$ denotes the norm of a bounded operator from $\mathfrak{H}$ to $\mathfrak{G}$. Sometimes we omit indices if this does not lead to confusion. By $I=I_{\mathfrak{H}}$ we denote the identity operator in $\mathfrak{H}$. If $\mathfrak{N}$ is a subspace in $\mathfrak{H}$, then $\mathfrak{N}^{\perp}:=\mathfrak{H} \ominus \mathfrak{N}$. If $P$ is the orthogonal projection of $\mathfrak{H}$ onto $\mathfrak{N}$, then we write $P^{\perp}$ for the 
orthogonal projection onto $\mathfrak{N}^{\perp}$. By $H^{s}(\mathcal{D}), s \in \mathbb{R}$, we denote the Sobolev spaces in the domain $\mathcal{D} \subset \mathbb{R}^{2}$.

0.5. The author is grateful to M. Sh. Birman for fruitful discussions.

\section{$\S 1$. Definition of the operator. Main Result}

1.1. Let $a>0$, and let

$$
\Pi:=\left\{\mathbf{x}=\left(x_{1}, x_{2}\right) \in \mathbb{R}^{2}: 0<x_{2}<a\right\} .
$$

We introduce the notation $\partial_{j}=\partial / \partial x_{j}, D_{j}=-i \partial_{j}, j=1,2$. Assume that $g(\mathbf{x})=$ $\operatorname{diag}\left\{g_{1}(\mathbf{x}), g_{2}(\mathbf{x})\right\}$, where the $g_{j}(\mathbf{x}), j=1,2$, are real-valued measurable functions in the strip $\Pi$ such that

$$
0<c_{0} \leq g_{j}(\mathbf{x}) \leq c_{1}<\infty, \quad \mathbf{x} \in \Pi, \quad j=1,2 .
$$

The functions $g_{j}\left(x_{1}, x_{2}\right)$ are assumed to be periodic in $x_{1}$ with period 1:

$$
g_{j}\left(x_{1}+1, x_{2}\right)=g_{j}\left(x_{1}, x_{2}\right), \quad \mathbf{x} \in \Pi, \quad j=1,2 .
$$

Next, suppose that the functions $g_{j}\left(x_{1}, x_{2}\right)$ are absolutely continuous with respect to the variable $x_{2} \in[0, a]$ (with $x_{1}$ fixed), and that

$$
\begin{gathered}
g_{j}\left(x_{1}, 0\right)=g_{j}\left(x_{1}, a\right), \quad x_{1} \in \mathbb{R}, \quad j=1,2, \\
\quad \underset{\mathbf{e s s} \sup }{\mathbf{x} \in \bar{\Pi}}\left|\partial_{2} g_{j}(\mathbf{x})\right| \leq c_{2}, \quad j=1,2 .
\end{gathered}
$$

In the space $L_{2}(\Pi)$, we consider the operator $A$ given formally by the differential expression

$$
\mathcal{A}=-\operatorname{div} g(\mathbf{x}) \operatorname{grad}=D_{1} g_{1}(\mathbf{x}) D_{1}+D_{2} g_{2}(\mathbf{x}) D_{2}
$$

with periodic boundary conditions in $x_{2}$. The precise definition of $A$ as a selfadjoint operator in $L_{2}(\Pi)$ is given in terms of the closed semibounded quadratic form

$$
a[u, u]=\int_{\Pi}\left(g_{1}(\mathbf{x})\left|D_{1} u\right|^{2}+g_{2}(\mathbf{x})\left|D_{2} u\right|^{2}\right) d \mathbf{x}, \quad u \in \widetilde{H}^{1}(\Pi) .
$$

Here $\widetilde{H}^{s}(\Pi), s \geq 0$, is the subspace in $H^{s}(\Pi)$ formed by all functions the $(a \mathbb{Z})$-periodic extension of which with respect to variable $x_{2}$ belongs to $H_{\text {loc }}^{s}\left(\mathbb{R}^{2}\right)$.

1.2. The homogenization problem consists in the study of the behavior of a periodic operator in the limit of small period. In $L_{2}(\Pi)$, we consider the operator $A_{\varepsilon}$ generated by the quadratic form

$$
\begin{array}{r}
a_{\varepsilon}[u, u]=\int_{\Pi}\left(g_{1}\left(x_{1} / \varepsilon, x_{2}\right)\left|D_{1} u\right|^{2}+g_{2}\left(x_{1} / \varepsilon, x_{2}\right)\left|D_{2} u\right|^{2}\right) d \mathbf{x}, \\
u \in \widetilde{H}^{1}(\Pi), \quad \varepsilon>0 .
\end{array}
$$

Our goal is to find the effective operator $A^{0}$ of the same form as $A$, but with coefficients independent of the "periodic" variable $x_{1}$ and such that

$$
\left(A_{\varepsilon}+I\right)^{-1} \rightarrow\left(A^{0}+I\right)^{-1}, \quad \varepsilon \rightarrow 0 .
$$

On the formal level, it is easily discovered that the effective operator $A^{0}$ is the operator in $L_{2}(\Pi)$ generated by the form

$$
a^{0}[u, u]=\int_{\Pi}\left(g_{1}^{0}\left(x_{2}\right)\left|D_{1} u\right|^{2}+g_{2}^{0}\left(x_{2}\right)\left|D_{2} u\right|^{2}\right) d \mathbf{x}, \quad u \in \widetilde{H}^{1}(\Pi),
$$


where the coefficients $g_{j}^{0}\left(x_{2}\right)$ are given by the formulas

$$
\begin{gathered}
g_{1}^{0}\left(x_{2}\right):=\left(\int_{0}^{1}\left(g_{1}\left(x_{1}, x_{2}\right)\right)^{-1} d x_{1}\right)^{-1}, \\
g_{2}^{0}\left(x_{2}\right):=\int_{0}^{1} g_{2}\left(x_{1}, x_{2}\right) d x_{1} .
\end{gathered}
$$

We note that conditions (1.1), (1.4) imply the following inequalities for the coefficients (1.7), (1.8):

$$
\begin{gathered}
c_{0} \leq g_{j}^{0}\left(x_{2}\right) \leq c_{1}, \quad x_{2} \in[0, a], \quad j=1,2, \\
\underset{x_{2} \in[0, a]}{\operatorname{ess} \sup _{2}}\left|\partial_{2} g_{1}^{0}\left(x_{2}\right)\right| \leq c_{2}\left(c_{1} / c_{0}\right)^{2}, \quad \underset{x_{2} \in[0, a]}{\operatorname{essup}}\left|\partial_{2} g_{2}^{0}\left(x_{2}\right)\right| \leq c_{2} .
\end{gathered}
$$

1.3. The main result of the paper is the following theorem.

Theorem 1.1. Suppose that conditions (1.1)-(1.4) are satisfied. Let $A_{\varepsilon}$ be the operator in $L_{2}(\Pi)$ that corresponds to the form (1.5), and let the operator $A^{0}$ correspond to the form (1.6), where the coefficients are defined by (1.7), (1.8). Then the following estimate is true:

$$
\left\|\left(A_{\varepsilon}+I\right)^{-1}-\left(A^{0}+I\right)^{-1}\right\|_{L_{2}(\Pi) \rightarrow L_{2}(\Pi)} \leq C \varepsilon, \quad 0<\varepsilon \leq 1 .
$$

The constant $C$ in (1.11) depends only on the constants $c_{0}$ and $c_{1}$ in (1.1) and on the constant $c_{2}$ in condition (1.4).

1.4. Interpolation. Let $\widetilde{H}^{-s}(\Pi), s \geq 0$, be the function space adjoint to $\widetilde{H}^{s}(\Pi)$ with respect to the $L_{2}(\Pi)$-duality. We estimate the resolvent $\left(A_{\varepsilon}+I\right)^{-1}$ as an operator from $\widetilde{H}^{-1}(\Pi)$ to $\widetilde{H}^{1}(\Pi)$. For $f \in \widetilde{H}^{-1}(\Pi)$, we put $u_{\varepsilon}=\left(A_{\varepsilon}+I\right)^{-1} f$. Then

$$
a_{\varepsilon}\left[u_{\varepsilon}, u_{\varepsilon}\right]+\left\|u_{\varepsilon}\right\|_{L_{2}(\Pi)}^{2}=\int_{\Pi} f \overline{u_{\varepsilon}} d \mathbf{x} .
$$

Combining this with the lower estimate (1.1), we arrive at the inequality

$$
\min \left\{c_{0}, 1\right\}\left\|u_{\varepsilon}\right\|_{H^{1}(\Pi)} \leq\|f\|_{\widetilde{H}^{-1}(\Pi)} .
$$

In other words,

$$
\left\|\left(A_{\varepsilon}+I\right)^{-1}\right\|_{\widetilde{H}^{-1}(\Pi) \rightarrow \widetilde{H}^{1}(\Pi)} \leq \max \left\{c_{0}^{-1}, 1\right\} .
$$

A similar estimate for $\left(A^{0}+I\right)^{-1}$ is also true. Consequently,

$$
\left\|\left(A_{\varepsilon}+I\right)^{-1}-\left(A^{0}+I\right)^{-1}\right\|_{\widetilde{H}^{-1}(\Pi) \rightarrow \widetilde{H}^{1}(\Pi)} \leq 2 \max \left\{c_{0}^{-1}, 1\right\} .
$$

Standard interpolation between (1.11) and (1.12) yields the following result.

Theorem 1.2. Under the conditions of Theorem 1.1, we have

$$
\left\|\left(A_{\varepsilon}+I\right)^{-1}-\left(A^{0}+I\right)^{-1}\right\|_{\widetilde{H}^{-s}(\Pi) \rightarrow \widetilde{H}^{s}(\Pi)} \leq C_{s} \varepsilon^{1-s}, \quad 0 \leq s<1, \quad 0<\varepsilon \leq 1 .
$$

Here $C_{s}=C^{1-s}\left(2 \max \left\{c_{0}^{-1}, 1\right\}\right)^{s}$. 


\section{§2. Reduction to operators on the CELL}

2.1. Direct integral expansion of the operator $A$ is obtained with the help of the Gelfand transformation $\mathcal{U}$. Putting $\Omega=(0,1) \times(0, a)$, we consider the Hilbert space

$$
\mathcal{K}=\int_{[-\pi, \pi)} \oplus L_{2}(\Omega) d k=L_{2}\left([-\pi, \pi) ; L_{2}(\Omega)\right)
$$

which is a direct integral with constant fibers. Initially, the operator $\mathcal{U}: L_{2}(\Pi) \rightarrow \mathcal{K}$ is defined on the functions $f \in C_{0}^{\infty}(\Pi)$ by the formula

$$
(\mathcal{U} f)(\mathbf{x}, k)=(2 \pi)^{-1 / 2} \sum_{m \in \mathbb{Z}} e^{-i k\left(x_{1}+m\right)} f\left(x_{1}+m, x_{2}\right), \quad \mathbf{x} \in \Omega, \quad k \in[-\pi, \pi),
$$

and then $\mathcal{U}$ extends by continuity to a unitary mapping from $L_{2}(\Pi)$ onto $\mathcal{K}$.

In $L_{2}(\Omega)$, we consider the operator $A(k)$ generated by the quadratic form

$$
a(k)[u, u]=\int_{\Omega}\left(g_{1}(\mathbf{x})\left|\left(D_{1}+k\right) u\right|^{2}+g_{2}(\mathbf{x})\left|D_{2} u\right|^{2}\right) d \mathbf{x}, \quad u \in \widetilde{H}^{1}(\Omega), \quad k \in \mathbb{R} .
$$

Here $\widetilde{H}^{1}(\Omega)$ is the subspace in $H^{1}(\Omega)$ formed by the functions the $(\mathbb{Z} \times(a \mathbb{Z}))$-periodic extensions of which belong to $H_{\text {loc }}^{1}\left(\mathbb{R}^{2}\right)$.

The operator $A$ is unitarily equivalent to the operator of multiplication by $A(k)$ in $\mathcal{K}$, which is expressed by the relation

$$
\mathcal{U} A \mathcal{U}^{-1}=\int_{[-\pi, \pi)} \oplus A(k) d k .
$$

2.2. We denote by $T_{\varepsilon}, \varepsilon>0$, the unitary scale transformation in $L_{2}(\Pi)$ defined by the formula

$$
\left(T_{\varepsilon} u\right)\left(y_{1}, x_{2}\right)=\varepsilon^{1 / 2} u\left(\varepsilon y_{1}, x_{2}\right), \quad y_{1} \in \mathbb{R}, \quad 0<x_{2}<a .
$$

Let $A(k, \varepsilon)$ be the operator in $L_{2}(\Omega)$ corresponding to the form

$$
\begin{array}{r}
a(k, \varepsilon)[u, u]=\int_{\Omega}\left(g_{1}(\mathbf{x})\left|\left(D_{1}+k\right) u\right|^{2}+\varepsilon^{2} g_{2}(\mathbf{x})\left|D_{2} u\right|^{2}\right) d \mathbf{x}, \\
u \in \widetilde{H}^{1}(\Omega), \quad k \in \mathbb{R}, \quad \varepsilon>0 .
\end{array}
$$

We put

$$
B(k, \varepsilon):=\varepsilon^{2}\left(A(k, \varepsilon)+\varepsilon^{2} I\right)^{-1}, \quad k \in \mathbb{R}, \quad \varepsilon>0 .
$$

The operator of multiplication by the operator-valued function (2.3) acting in the direct integral (2.1) is denoted by $B_{\varepsilon}$ :

$$
B_{\varepsilon}:=\int_{[-\pi, \pi)} \oplus B(k, \varepsilon) d k, \quad \varepsilon>0 .
$$

It is easily seen [7, (5.6)] that

$$
\left(A_{\varepsilon}+I\right)^{-1}=T_{\varepsilon}^{*} \mathcal{U}^{*} B_{\varepsilon} \mathcal{U} T_{\varepsilon}, \quad \varepsilon>0 .
$$

Relation (2.4) reduces the problem to the study of the operator family (2.3) acting in $L_{2}(\Omega)$. 
2.3. Let $A^{0}(k, \varepsilon)$ be the operator in $L_{2}(\Omega)$ corresponding to the form

$$
a^{0}(k, \varepsilon)[u, u]=\int_{\Omega}\left(g_{1}^{0}\left(x_{2}\right)\left|\left(D_{1}+k\right) u\right|^{2}+\varepsilon^{2} g_{2}^{0}\left(x_{2}\right)\left|D_{2} u\right|^{2}\right) d \mathbf{x}, \quad u \in \widetilde{H}^{1}(\Omega) .
$$

We put

$$
\begin{gathered}
B^{0}(k, \varepsilon):=\varepsilon^{2}\left(A^{0}(k, \varepsilon)+\varepsilon^{2} I\right)^{-1}, \quad \varepsilon>0, \\
B_{\varepsilon}^{0}:=\int_{[-\pi, \pi)} \oplus B^{0}(k, \varepsilon) d k, \quad \varepsilon>0 .
\end{gathered}
$$

As in (2.4), we have

$$
\left(A^{0}+I\right)^{-1}=T_{\varepsilon}^{*} \mathcal{U}^{*} B_{\varepsilon}^{0} \mathcal{U} T_{\varepsilon}, \quad \varepsilon>0 .
$$

Theorem 1.1 is a direct consequence of relations (2.4), (2.6) and the following theorem.

Theorem 2.1. Suppose that conditions (1.1), (1.3), and (1.4) are satisfied. Let $B(k, \varepsilon)$ and $B^{0}(k, \varepsilon)$ be the operators defined by $(2.3)$ and $(2.5)$. Then

$$
\max _{k \in[-\pi, \pi)}\left\|B(k, \varepsilon)-B^{0}(k, \varepsilon)\right\|_{L_{2}(\Omega) \rightarrow L_{2}(\Omega)} \leq C \varepsilon, \quad 0<\varepsilon \leq 1 .
$$

The constant $C$ in (2.7) depends only on the constants $c_{0}$ and $c_{1}$ in (1.1) and on the constant $c_{2}$ in (1.4).

\section{§3. The operator Pencil $A_{1}(k)$}

3.1. If we formally put $\varepsilon=0$, then the operator $A(k, \varepsilon)$ defined in Subsection 2.2 will turn into the operator $A_{1}(k)$ given by the expression $\left(D_{1}+k\right) g_{1}(\mathbf{x})\left(D_{1}+k\right)$ and periodic boundary conditions in $x_{1}$, and the boundary conditions in $x_{2}$ "disappear". Strictly speaking, $A_{1}(k)$ is the selfadjoint operator in $L_{2}(\Omega)$ generated by the quadratic form

$$
\begin{gathered}
a_{1}(k)[u, u]=\int_{\Omega} g_{1}(\mathbf{x})\left|\left(D_{1}+k\right) u\right|^{2} d \mathbf{x}, \\
\operatorname{Dom} a_{1}(k)=\int_{(0, a)} \oplus \widetilde{H}^{1}(0,1) d x_{2}=L_{2}\left((0, a) ; \widetilde{H}^{1}(0,1)\right) .
\end{gathered}
$$

Here $\widetilde{H}^{1}(0,1)$ is the subspace in $H^{1}(0,1)$ formed by the functions whose $\mathbb{Z}$-periodic extensions belong to $H_{\mathrm{loc}}^{1}(\mathbb{R})$.

In this section we study the operator pencil $A_{1}(k)$. Here, it suffices to impose only condition (1.1) with $j=1$. We shall not use conditions (1.2)-(1.4). Now it is convenient to interpret the space $L_{2}(\Omega)$ as a direct integral in $x_{2}$ with constant fibers $L_{2}(0,1)$ :

$$
L_{2}(\Omega)=\int_{(0, a)} \oplus L_{2}(0,1) d x_{2} .
$$

The operator $A_{1}(k)$ acts in the direct integral (3.3) "layerwise", i.e., it is the operator of multiplication by the operator-valued function $A_{1}\left(k ; x_{2}\right)$ :

$$
A_{1}(k)=\int_{(0, a)} \oplus A_{1}\left(k ; x_{2}\right) d x_{2},
$$

where $A_{1}\left(k ; x_{2}\right)$ is the selfadjoint operator in $L_{2}(0,1)$ generated by the form

$$
a_{1}\left(k ; x_{2}\right)[v, v]=\int_{0}^{1} g_{1}\left(x_{1}, x_{2}\right)\left|\left(D_{1}+k\right) v\right|^{2} d x_{1}, \quad v \in \widetilde{H}^{1}(0,1), x_{2} \in(0, a) .
$$


3.2. The method of the papers $\left[7,8\right.$, is applicable to the operator family $A_{1}\left(k ; x_{2}\right)$ acting in $L_{2}(0,1)$. This family admits the following factorization:

$$
\begin{gathered}
A_{1}\left(k ; x_{2}\right)=\left(X\left(k ; x_{2}\right)\right)^{*} X\left(k ; x_{2}\right), \\
X\left(k ; x_{2}\right)=X_{0}\left(x_{2}\right)+k X_{1}\left(x_{2}\right),
\end{gathered}
$$

where $X_{0}\left(x_{2}\right)$ is the (closed) operator in $L_{2}(0,1)$ given by the expression $\left(g_{1}\left(x_{1}, x_{2}\right)\right)^{\frac{1}{2}} D_{1}$ on the domain $\widetilde{H}^{1}(0,1)$, and $X_{1}\left(x_{2}\right)$ is the (bounded) operator of multiplication by the function $\left(g_{1}\left(x_{1}, x_{2}\right)\right)^{1 / 2}$. Then the operator $(3.7)$ is closed in $L_{2}(0,1)$ on the domain $\widetilde{H}^{1}(0,1)$. Obviously, the kernel

$$
\widetilde{\mathfrak{N}}=\operatorname{Ker} A_{1}\left(0 ; x_{2}\right)=\operatorname{Ker} X_{0}\left(x_{2}\right)=\left\{u \in L_{2}(0,1): u=\text { const }\right\}
$$

is one-dimensional. Let $d_{0}\left(x_{2}\right)$ denote the distance between the point $\lambda=0$ and the rest of the spectrum of $A_{1}\left(0 ; x_{2}\right)$. We estimate $d_{0}\left(x_{2}\right)$ from below.

By condition (1.1) (with $j=1$ ), the quadratic form (3.5) with $k=0$ satisfies the estimate

$$
a_{1}\left(0 ; x_{2}\right)[v, v] \geq c_{0} \int_{0}^{1}\left|D_{1} v\left(x_{1}\right)\right|^{2} d x_{1}, \quad v \in \widetilde{H}^{1}(0,1) .
$$

Expanding the function $v \in \widetilde{H}^{1}(0,1)$ in the Fourier series

$$
v\left(x_{1}\right)=\sum_{m \in \mathbb{Z}} \widehat{v}_{m} e^{2 \pi i m x_{1}}
$$

and using (3.9), we obtain

$$
a_{1}\left(0 ; x_{2}\right)[v, v] \geq c_{0} \sum_{m \in \mathbb{Z}} 4 \pi^{2} m^{2}\left|\widehat{v}_{m}\right|^{2}, \quad v \in \widetilde{H}^{1}(0,1) .
$$

If $v \perp \widetilde{\mathfrak{N}}$ in $L_{2}(0,1)$, then $\widehat{v}_{0}=0$, whence

$$
a_{1}\left(0 ; x_{2}\right)[v, v] \geq 4 \pi^{2} c_{0}\|v\|_{L_{2}(0,1)}^{2}, \quad v \in \widetilde{H}^{1}(0,1), v \perp \widetilde{\mathfrak{N}} .
$$

This implies the inequality

$$
d_{0}\left(x_{2}\right) \geq d_{0}:=4 \pi^{2} c_{0}
$$

In accordance with [7] $\S 1$, we choose $\delta$ such that

$$
0<\delta<d_{0} / 8=\pi^{2} c_{0} / 2 \text {. }
$$

For instance, we can put

$$
\delta=\pi^{2} c_{0} / 4
$$

Next, we estimate the norm of the operator $X_{1}\left(x_{2}\right)$. By the upper estimate (1.1) (with $j=1$ ), we have

$$
\left\|X_{1}\left(x_{2}\right)\right\|_{L_{2}(0,1) \rightarrow L_{2}(0,1)} \leq \sup _{0<x_{1}<1}\left(g_{1}\left(x_{1}, x_{2}\right)\right)^{1 / 2} \leq c_{1}^{1 / 2}
$$

for all $x_{2} \in(0, a)$. As in [7, (1.5)], we choose a number $t_{0}=t_{0}(\delta)$ such that $t_{0}(\delta) \leq$ $\delta^{1 / 2}\left\|X_{1}\left(x_{2}\right)\right\|^{-1}$ for all $x_{2} \in(0, a)$. It suffices to put

$$
t_{0}=\delta^{1 / 2} c_{1}^{-1 / 2}=\pi c_{0}^{1 / 2} / 2 c_{1}^{1 / 2} .
$$

Let $F\left(k ; x_{2} ; \sigma\right)$ be the spectral projection of the operator $A_{1}\left(k ; x_{2}\right)$ corresponding to the closed interval $[0, \sigma]$. From [7, Proposition 1.2] it follows that

$$
\operatorname{dim} F\left(k ; x_{2} ; \delta\right) L_{2}(0,1)=\operatorname{dim} F\left(k ; x_{2} ; 3 \delta\right) L_{2}(0,1)=1, \quad|k|=t \leq t_{0} .
$$

Therefore, for all $x_{2} \in(0, a)$ the interval $(\delta, 3 \delta)$ is free of the spectrum of the operator $A_{1}\left(k ; x_{2}\right)$ if $|k| \leq t_{0}$. We put $F\left(k ; x_{2}\right):=F\left(k ; x_{2} ; \delta\right)$. 
3.3. The spectral germ $S\left(x_{2}\right)$ of the operator family $A_{1}\left(k ; x_{2}\right)$. In accordance with [7, 8], we introduce the operator $S\left(x_{2}\right)$ acting in the one-dimensional space $\widetilde{\mathfrak{N}}$ (see (3.8)) and called the spectral germ of the operator family $A_{1}\left(k ; x_{2}\right)$ at $k=0$. In our case (i.e., for the one-dimensional elliptic operator of the form $\left.-\frac{d}{d x} g(x) \frac{d}{d x}\right)$, the germ is calculated explicitly (see [7, Subsection 6.1]): the operator $S\left(x_{2}\right)$ is multiplication by the number $g_{1}^{0}\left(x_{2}\right)$ (see (1.7)).

Let $\widetilde{P}$ be the orthoprojection in $L_{2}(0,1)$ onto the one-dimensional subspace $\widetilde{\mathfrak{N}}$. By [7. (2.13)-(2.15)], the following approximations are valid:

$$
\begin{gathered}
F\left(k ; x_{2}\right)=\widetilde{P}+t \Phi\left(k ; x_{2}\right), \quad t=|k| \leq t_{0}, \quad x_{2} \in(0, a), \\
A_{1}\left(k ; x_{2}\right) F\left(k ; x_{2}\right)=t^{2} S\left(x_{2}\right) \widetilde{P}+t^{3} \Psi\left(k ; x_{2}\right), \quad t=|k| \leq t_{0}, \quad x_{2} \in(0, a) .
\end{gathered}
$$

Here, the operators $\Phi\left(k ; x_{2}\right)$ and $\Psi\left(k ; x_{2}\right)$ admit the estimates

$$
\begin{aligned}
& \left\|\Phi\left(k ; x_{2}\right)\right\|_{L_{2}(0,1) \rightarrow L_{2}(0,1)} \leq C_{1}, \quad t=|k| \leq t_{0}, \quad x_{2} \in(0, a), \\
& \left\|\Psi\left(k ; x_{2}\right)\right\|_{L_{2}(0,1) \rightarrow L_{2}(0,1)} \leq C_{2}, \quad t=|k| \leq t_{0}, \quad x_{2} \in(0, a) .
\end{aligned}
$$

The constants $C_{1}, C_{2}$ are controlled explicitly. By [7 (1.4.3), (1.4.6)], and (3.10), we have

$$
\begin{aligned}
& C_{1}=\beta_{1} \delta^{-1 / 2} \max _{x_{2} \in(0, a)}\left\|X_{1}\left(x_{2}\right)\right\| \leq \beta_{1} \delta^{-1 / 2} c_{1}^{1 / 2}, \\
& C_{2}=\beta_{2} \delta^{-1 / 2} \max _{x_{2} \in(0, a)}\left\|X_{1}\left(x_{2}\right)\right\|^{3} \leq \beta_{2} \delta^{-1 / 2} c_{1}^{3 / 2},
\end{aligned}
$$

where $\beta_{1}$ and $\beta_{2}$ are absolute constants.

3.4. The spectral germ of the operator family $A_{1}(k)$. We return to the family (3.4) in $L_{2}(\Omega)$. First, we note that the interval $(\delta, 3 \delta)$ is free of the spectrum of $A_{1}(k)$ for $|k| \leq t_{0}$ (this follows from (3.4) and (3.12)).

By (3.4) and (3.6), we have

$$
A_{1}(k)=X(k)^{*} X(k)
$$

where

$$
X(k)=\int_{(0, a)} \oplus X\left(k ; x_{2}\right) d x_{2} .
$$

Here, by (3.7),

$$
\begin{aligned}
X(k) & =X_{0}+k X_{1}, \\
X_{0} & :=\int_{(0, a)} \oplus X_{0}\left(x_{2}\right) d x_{2}, \\
X_{1} & :=\int_{(0, a)} \oplus X_{1}\left(x_{2}\right) d x_{2} .
\end{aligned}
$$

Clearly, $X_{0}$ is the operator in $L_{2}(\Omega)$ given by the expression $\left(g_{1}(\mathbf{x})\right)^{1 / 2} D_{1}$ on the domain (3.2), and $X_{1}$ is the operator of multiplication by the function $\left(g_{1}(\mathbf{x})\right)^{1 / 2}$.

For the operator $A_{1}(0)$ with $k=0$, we have

$$
\mathfrak{N}=\operatorname{Ker} A_{1}(0)=\operatorname{Ker} X_{0}=\left\{u \in L_{2}(\Omega): u=u\left(x_{2}\right)\right\} .
$$

Thus, the kernel $\mathfrak{N}$ is isomorphic to $L_{2}(0, a)$ and consists of functions depending only on the second variable. Obviously,

$$
\mathfrak{N}=\int_{(0, a)} \oplus \widetilde{\mathfrak{N}} d x_{2}
$$


which means that the space $\mathfrak{N}\left(=L_{2}(0, a)\right)$ can be viewed as a direct integral of the one-dimensional spaces $\widetilde{\mathfrak{N}}(=\mathbb{C})$. It is also clear that the orthoprojection $P$ of $L_{2}(\Omega)$ onto the kernel $\mathfrak{N}$ can be represented as the direct integral of the orthoprojections $\widetilde{P}$ of $L_{2}(0,1)$ onto $\widetilde{\mathfrak{N}}$ :

$$
P=\int_{(0, a)} \oplus \widetilde{P} d x_{2}
$$

In the space (3.18), we introduce the decomposable operator

$$
S=\int_{(0, a)} \oplus S\left(x_{2}\right) d x_{2},
$$

which is the operator of multiplication by the function $g_{1}^{0}\left(x_{2}\right)$. The operator $S$ is called the spectral germ of the operator family $A_{1}(k)$ at $k=0$.

Let $F(k)$ denote the spectral projection of the operator $A_{1}(k)$ corresponding to the interval $[0, \delta]$. Since $A_{1}(k)$ can be represented as the direct integral (3.4), we have

$$
F(k)=\int_{(0, a)} \oplus F\left(k ; x_{2}\right) d x_{2} .
$$

From (3.13), (3.19), and (3.20) it follows that

$$
\begin{gathered}
F(k)=P+t \Phi(k), \quad t=|k| \leq t_{0}, \\
\Phi(k)=\int_{(0, a)} \oplus \Phi\left(k ; x_{2}\right) d x_{2} .
\end{gathered}
$$

Similarly, (3.4), (3.14), (3.19), and (3.20) imply the relations

$$
\begin{gathered}
A_{1}(k) F(k)=t^{2} S P+t^{3} \Psi(k), \quad t=|k| \leq t_{0}, \\
\Psi(k)=\int_{(0, a)} \oplus \Psi\left(k ; x_{2}\right) d x_{2} .
\end{gathered}
$$

By (3.15) and (3.16), the operators (3.22) and (3.24) satisfy the estimates

$$
\begin{aligned}
& \|\Phi(k)\|_{L_{2}(\Omega) \rightarrow L_{2}(\Omega)} \leq C_{1}, \quad t=|k| \leq t_{0}, \\
& \|\Psi(k)\|_{L_{2}(\Omega) \rightarrow L_{2}(\Omega)} \leq C_{2}, \quad t=|k| \leq t_{0} .
\end{aligned}
$$

\section{$\S 4$. Approximation of the operator family $B(k ; \varepsilon)$}

4.1. Now we return to the study of the operator family $A(k, \varepsilon)$ introduced in Subsection

2.2. Our goal is to approximate the operator $B(k, \varepsilon)$ defined by $(2.3)$.

In the space $\mathfrak{N}=L_{2}(0, a)$ (see (3.17)), we consider the quadratic form

$$
\begin{gathered}
s(k, \varepsilon)[\omega, \omega]=\int_{0}^{a}\left(g_{1}^{0}\left(x_{2}\right) t^{2}\left|\omega\left(x_{2}\right)\right|^{2}+g_{2}^{0}\left(x_{2}\right) \varepsilon^{2}\left|D_{2} \omega\left(x_{2}\right)\right|^{2}\right) d x_{2}, \\
k \in[-\pi, \pi), \quad \varepsilon>0, \\
\operatorname{Dom} s(k, \varepsilon)=\widetilde{H}^{1}(0, a) .
\end{gathered}
$$

Let $\widetilde{H}^{s}(0, a)$ denote the subspace in $H^{s}(0, a)$ formed by the functions the $(a \mathbb{Z})$-periodic extensions of which belong to $H_{\mathrm{loc}}^{s}(\mathbb{R})$. The form (4.1) is closed and semibounded on the domain (4.2). The selfadjoint operator in $L_{2}(0, a)$ corresponding to this form is denoted by $S(k, \varepsilon)$. Clearly, under conditions (1.9) and (1.10), the operator $S(k, \varepsilon)$ is given by the differential expression

$$
S(k, \varepsilon)=g_{1}^{0}\left(x_{2}\right) t^{2}+\varepsilon^{2} D_{2} g_{2}^{0}\left(x_{2}\right) D_{2}
$$


on the domain

$$
\operatorname{Dom} S(k, \varepsilon)=\widetilde{H}^{2}(0, a) .
$$

Note that the first summand in (4.3) corresponds to the operator $t^{2} S$.

The key role in the proof of Theorem 2.1 is played by the following theorem.

Theorem 4.1. Suppose that conditions (1.1), (1.3), and (1.4) are satisfied. Let $P$ be the orthoprojection of $L_{2}(\Omega)$ onto the subspace $\mathfrak{N}$ of functions depending only on $x_{2}$. Let $A(k, \varepsilon)$ be the operator in $L_{2}(\Omega)$ corresponding to the quadratic form $(2.2)$, and let $S(k, \varepsilon)$ be the operator in $\mathfrak{N}$ defined by (4.3) and (4.4). Then

$$
\begin{array}{r}
\varepsilon^{2}\left\|\left(A(k, \varepsilon)+\varepsilon^{2} I\right)^{-1}-\left(S(k, \varepsilon) P+\varepsilon^{2} I\right)^{-1} P\right\|_{L_{2}(\Omega) \rightarrow L_{2}(\Omega)} \leq \widetilde{C} \varepsilon, \\
|k| \leq t_{0}, \quad 0<\varepsilon \leq 1 .
\end{array}
$$

The number $t_{0}$ is defined as in (3.11). The constant $\widetilde{C}$ depends only on the constants $c_{0}$ and $c_{1}$ in (1.1) and on the constant $c_{2}$ in (1.4).

4.2. Deduction of Theorem 2.1 from Theorem 4.1. Let $A^{0}(k, \varepsilon)$ be the operator family corresponding to the effective operator $A^{0}$ defined in Subsection 2.3. For $A^{0}(k, \varepsilon)$, we define the corresponding operator $S^{0}(k, \varepsilon)$ by the same rule as for $A(k, \varepsilon)$. Clearly, $S^{0}(k, \varepsilon)=S(k, \varepsilon)$. Applying Theorem 4.1 to the operator $A^{0}(k, \varepsilon)$, we obtain

$$
\begin{array}{r}
\varepsilon^{2}\left\|\left(A^{0}(k, \varepsilon)+\varepsilon^{2} I\right)^{-1}-\left(S(k, \varepsilon) P+\varepsilon^{2} I\right)^{-1} P\right\|_{L_{2}(\Omega) \rightarrow L_{2}(\Omega)} \leq \widetilde{C}^{\prime} \varepsilon, \\
|k| \leq t_{0}, \quad 0<\varepsilon \leq 1 .
\end{array}
$$

Since the coefficients $g_{j}^{0}\left(x_{2}\right), j=1,2$, satisfy estimate (1.9) with the same constants $c_{0}$ and $c_{1}$ as in (1.1), the number $t_{0}$ (see (3.11)) in (4.5) and in (4.6) is one and the same. Moreover, combining (1.9) and (1.10) with the statement of Theorem 4.1 concerning the constant $\widetilde{C}$, we see that the constant $\widetilde{C}^{\prime}$ in (4.6) depends only on $c_{0}, c_{1}$, and $c_{2}$.

From (4.5) and (4.6) it follows that

$$
\begin{array}{r}
\varepsilon^{2}\left\|\left(A(k, \varepsilon)+\varepsilon^{2} I\right)^{-1}-\left(A^{0}(k, \varepsilon)+\varepsilon^{2} I\right)^{-1}\right\|_{L_{2}(\Omega) \rightarrow L_{2}(\Omega)} \leq\left(\widetilde{C}+\widetilde{C}^{\prime}\right) \varepsilon, \\
|k| \leq t_{0}, \quad 0<\varepsilon \leq 1 .
\end{array}
$$

For $|k|>t_{0}$, the required estimate can easily be obtained by variational arguments. By (1.1) and (2.2), we have

$$
\begin{array}{r}
a(k, \varepsilon)[u, u] \geq c_{0} \int_{\Omega}\left(\left|\left(D_{1}+k\right) u\right|^{2}+\varepsilon^{2}\left|D_{2} u\right|^{2}\right) d \mathbf{x}, \\
u \in \widetilde{H}^{1}(\Omega), \quad k \in[-\pi, \pi), \quad \varepsilon>0 .
\end{array}
$$

Expanding the function $u \in \widetilde{H}^{1}(\Omega)$ in the Fourier series with respect to variable $x_{1}$,

$$
u\left(x_{1}, x_{2}\right)=\sum_{m \in \mathbb{Z}} \widehat{u}_{m}\left(x_{2}\right) e^{2 \pi i m x_{1}},
$$

we obtain

$$
\begin{array}{r}
a(k, \varepsilon)[u, u] \geq c_{0} \sum_{m \in \mathbb{Z}}(2 \pi m+k)^{2} \int_{0}^{a}\left|\widehat{u}_{m}\left(x_{2}\right)\right|^{2} d x_{2}, \\
u \in \widetilde{H}^{1}(\Omega), \quad k \in[-\pi, \pi), \quad \varepsilon>0 .
\end{array}
$$

Obviously, if $k \in\left[-\pi,-t_{0}\right] \cup\left[t_{0}, \pi\right)$ (see (3.11)), then

$$
|2 \pi m+k| \geq t_{0}, \quad m \in \mathbb{Z} .
$$

Then (4.9) implies that

$$
a(k, \varepsilon)[u, u] \geq c_{0} t_{0}^{2}\|u\|_{L_{2}(\Omega)}^{2}, \quad u \in \widetilde{H}^{1}(\Omega), \quad k \in\left[-\pi,-t_{0}\right] \cup\left[t_{0}, \pi\right), \quad \varepsilon>0 .
$$


Consequently,

$$
\left\|\left(A(k, \varepsilon)+\varepsilon^{2} I\right)^{-1}\right\|_{L_{2}(\Omega) \rightarrow L_{2}(\Omega)} \leq c_{0}^{-1} t_{0}^{-2}, \quad k \in\left[-\pi,-t_{0}\right] \cup\left[t_{0}, \pi\right), \quad \varepsilon>0 .
$$

The same estimate is true with $A(k, \varepsilon)$ replaced by $A^{0}(k, \varepsilon)$. Combining this with (4.7), we obtain (2.7) with the constant $C=\max \left\{\widetilde{C}+\widetilde{C}^{\prime}, 2 c_{0}^{-1} t_{0}^{-2}\right\}$ depending only on $c_{0}, c_{1}$, and $c_{2}$.

Theorem 4.1 is proved in $\S \S 5-7$.

\section{§5. Preliminary estimates}

5.1. By (4.9), we have

$$
\begin{array}{r}
a(k, \varepsilon)[u, u]+\varepsilon^{2}\|u\|_{L_{2}(\Omega)}^{2} \geq\left(c_{0} t^{2}+\varepsilon^{2}\right)\|u\|_{L_{2}(\Omega)}^{2} \geq c_{*}\left(t^{2}+\varepsilon^{2}\right)\|u\|_{L_{2}(\Omega)}^{2}, \\
u \in \widetilde{H}^{1}(\Omega), \quad k \in[-\pi, \pi), \quad \varepsilon>0,
\end{array}
$$

where $c_{*}=\min \left\{c_{0}, 1\right\}$.

As in (4.8), from (1.1) and (3.1) it follows that

$$
a_{1}(k)[u, u] \geq c_{0} \int_{\Omega}\left|\left(D_{1}+k\right) u\right|^{2} d \mathbf{x}, \quad u \in \widetilde{H}^{1}(\Omega), \quad k \in[-\pi, \pi),
$$

whence

$$
\begin{array}{r}
a_{1}(k)[u, u]+\varepsilon^{2}\|u\|_{L_{2}(\Omega)}^{2} \geq\left(c_{0} t^{2}+\varepsilon^{2}\right)\|u\|_{L_{2}(\Omega)}^{2} \geq c_{*}\left(t^{2}+\varepsilon^{2}\right)\|u\|_{L_{2}(\Omega)}^{2}, \\
u \in \operatorname{Dom} a_{1}(k), \quad k \in[-\pi, \pi), \quad \varepsilon>0 .
\end{array}
$$

By (1.9), the form (4.1) satisfies the estimate

$$
\begin{aligned}
s(k, \varepsilon)[\omega, \omega] & \geq c_{0} \int_{0}^{a}\left(t^{2}|\omega|^{2}+\varepsilon^{2}\left|D_{2} \omega\right|^{2}\right) d x_{2}, \\
\omega & \in \widetilde{H}^{1}(0, a), \quad k \in[-\pi, \pi), \quad \varepsilon>0 .
\end{aligned}
$$

Then

$$
\begin{array}{r}
s(k, \varepsilon)[\omega, \omega]+\varepsilon^{2}\|\omega\|_{L_{2}(0, a)}^{2} \geq\left(c_{0} t^{2}+\varepsilon^{2}\right)\|\omega\|_{L_{2}(0, a)}^{2} \geq c_{*}\left(t^{2}+\varepsilon^{2}\right)\|\omega\|_{L_{2}(0, a)}^{2}, \\
\omega \in \widetilde{H}^{1}(0, a), \quad k \in[-\pi, \pi), \quad \varepsilon>0 .
\end{array}
$$

Relations (5.1), (5.3), and (5.5) imply that

$$
\begin{gathered}
\left\|\left(A(k, \varepsilon)+\varepsilon^{2} I\right)^{-1}\right\|_{L_{2}(\Omega) \rightarrow L_{2}(\Omega)} \leq c_{*}^{-1}\left(t^{2}+\varepsilon^{2}\right)^{-1}, \\
\left\|\left(A_{1}(k)+\varepsilon^{2} I\right)^{-1}\right\|_{L_{2}(\Omega) \rightarrow L_{2}(\Omega)} \leq c_{*}^{-1}\left(t^{2}+\varepsilon^{2}\right)^{-1}, \\
\left\|\left(S(k, \varepsilon) P+\varepsilon^{2} I\right)^{-1} P\right\|_{L_{2}(\Omega) \rightarrow L_{2}(\Omega)} \leq c_{*}^{-1}\left(t^{2}+\varepsilon^{2}\right)^{-1}
\end{gathered}
$$

for all $k \in[-\pi, \pi)$ and $\varepsilon \geq 0$.

Next, from the estimate (see (4.8))

$$
\begin{aligned}
& c_{0} \int_{\Omega}\left(\left|\left(D_{1}+k\right) u\right|^{2}+\varepsilon^{2}\left|D_{2} u\right|^{2}\right) d \mathbf{x} \\
& \quad \leq a(k, \varepsilon)[u, u]+\varepsilon^{2}\|u\|_{L_{2}(\Omega)}^{2}=\left\|\left(A(k, \varepsilon)+\varepsilon^{2} I\right)^{1 / 2} u\right\|_{L_{2}(\Omega)}^{2}, \quad u \in \widetilde{H}^{1}(\Omega),
\end{aligned}
$$

it follows that

$$
\begin{gathered}
\left\|\left(D_{1}+k\right)\left(A(k, \varepsilon)+\varepsilon^{2} I\right)^{-1 / 2}\right\|_{L_{2}(\Omega) \rightarrow L_{2}(\Omega)} \leq c_{0}^{-1 / 2}, \\
\left\|\varepsilon D_{2}\left(A(k, \varepsilon)+\varepsilon^{2} I\right)^{-1 / 2}\right\|_{L_{2}(\Omega) \rightarrow L_{2}(\Omega)} \leq c_{0}^{-1 / 2}
\end{gathered}
$$

for all $k \in[-\pi, \pi)$ and $\varepsilon>0$. Similarly, (5.2) implies

$$
\left\|\left(D_{1}+k\right) A_{1}(k)^{-1 / 2}\right\|_{L_{2}(\Omega) \rightarrow L_{2}(\Omega)} \leq c_{0}^{-1 / 2}, \quad k \in[-\pi, \pi) .
$$


Finally, from (5.4) we obtain

$$
\left\|\varepsilon D_{2}\left(S(k, \varepsilon) P+\varepsilon^{2} I\right)^{-1 / 2} P\right\|_{L_{2}(\Omega) \rightarrow L_{2}(\Omega)} \leq c_{0}^{-1 / 2}
$$

for all $k \in[-\pi, \pi)$ and $\varepsilon>0$.

5.2. Recall that $F(k)$ denotes the spectral projection of the operator $A_{1}(k)$ for the interval $[0, \delta]$, and $P$ denotes the orthoprojection of $L_{2}(\Omega)$ onto the subspace $\mathfrak{N}$ of functions depending only on $x_{2}$.

Lemma 5.1. Let $t=|k| \leq t_{0}, \varepsilon>0$. Then

$$
\begin{gathered}
\left\|F(k)^{\perp}\left(A(k, \varepsilon)+\varepsilon^{2} I\right)^{-1 / 2}\right\|_{L_{2}(\Omega) \rightarrow L_{2}(\Omega)} \\
=\left\|\left(A(k, \varepsilon)+\varepsilon^{2} I\right)^{-1 / 2} F(k)^{\perp}\right\|_{L_{2}(\Omega) \rightarrow L_{2}(\Omega)} \leq \delta^{-1 / 2} ; \\
\left\|\left(A(k, \varepsilon)+\varepsilon^{2} I\right)^{-1 / 2} P^{\perp}\right\|_{L_{2}(\Omega) \rightarrow L_{2}(\Omega)} \leq C_{3}:=\delta^{-1 / 2}+C_{1} c_{*}^{-1 / 2} ; \\
\left\|F(k)^{\perp}\left(S(k, \varepsilon) P+\varepsilon^{2} I\right)^{-1 / 2} P\right\|_{L_{2}(\Omega) \rightarrow L_{2}(\Omega)} \leq C_{4}:=C_{1} c_{*}^{-1 / 2} .
\end{gathered}
$$

Proof. By (2.2) and (3.1), we have

$$
a_{1}(k)[u, u]+\varepsilon^{2}\|u\|_{L_{2}(\Omega)}^{2} \leq a(k, \varepsilon)[u, u]+\varepsilon^{2}\|u\|_{L_{2}(\Omega)}^{2}, \quad u \in \widetilde{H}^{1}(\Omega) .
$$

Consequently,

$$
\left\|\left(A_{1}(k)+\varepsilon^{2} I\right)^{1 / 2}\left(A(k, \varepsilon)+\varepsilon^{2} I\right)^{-1 / 2}\right\|_{L_{2}(\Omega) \rightarrow L_{2}(\Omega)} \leq 1 .
$$

Therefore,

$$
\begin{aligned}
\| F(k)^{\perp} & \left(A(k, \varepsilon)+\varepsilon^{2} I\right)^{-1 / 2} \|_{L_{2}(\Omega) \rightarrow L_{2}(\Omega)} \\
\leq & \left\|F(k)^{\perp}\left(A_{1}(k)+\varepsilon^{2} I\right)^{-1 / 2}\right\|_{L_{2}(\Omega) \rightarrow L_{2}(\Omega)} \\
& \times\left\|\left(A_{1}(k)+\varepsilon^{2} I\right)^{1 / 2}\left(A(k, \varepsilon)+\varepsilon^{2} I\right)^{-1 / 2}\right\|_{L_{2}(\Omega) \rightarrow L_{2}(\Omega)} \\
\leq & \delta^{-1 / 2}
\end{aligned}
$$

which proves (5.12).

In order to prove (5.13), we use (5.12), (5.6) and (3.21), (3.25):

$$
\begin{aligned}
& \left\|\left(A(k, \varepsilon)+\varepsilon^{2} I\right)^{-1 / 2} P^{\perp}\right\|_{L_{2}(\Omega) \rightarrow L_{2}(\Omega)} \\
& \quad=\left\|\left(A(k, \varepsilon)+\varepsilon^{2} I\right)^{-1 / 2}\left(F(k)^{\perp}+t \Phi(k)\right)\right\|_{L_{2}(\Omega) \rightarrow L_{2}(\Omega)} \\
& \quad \leq \delta^{-1 / 2}+C_{1} c_{*}^{-1 / 2} t\left(t^{2}+\varepsilon^{2}\right)^{-1 / 2} \leq C_{3} .
\end{aligned}
$$

Inequality (5.14) follows from (3.21), (3.25), (5.7), and the obvious relation

$$
P^{\perp}\left(S(k, \varepsilon) P+\varepsilon^{2} I\right)^{-1 / 2} P=0 .
$$

We have

$$
\begin{aligned}
& \left\|F(k)^{\perp}\left(S(k, \varepsilon) P+\varepsilon^{2} I\right)^{-1 / 2} P\right\|_{L_{2}(\Omega) \rightarrow L_{2}(\Omega)} \\
& \quad=\left\|t \Phi(k)\left(S(k, \varepsilon) P+\varepsilon^{2} I\right)^{-1 / 2} P\right\|_{L_{2}(\Omega) \rightarrow L_{2}(\Omega)} \\
& \quad \leq C_{1} c_{*}^{-1 / 2} t\left(t^{2}+\varepsilon^{2}\right)^{-1 / 2} \leq C_{4} . \quad \square
\end{aligned}
$$

Lemma 5.1 and inequalities (5.6) and (5.7) imply the following statement.

Corollary 5.2. Let $t=|k| \leq t_{0}, \varepsilon>0$. Then

$$
\begin{gathered}
\varepsilon^{2}\left\|\left(A(k, \varepsilon)+\varepsilon^{2} I\right)^{-1} P^{\perp}\right\|_{L_{2}(\Omega) \rightarrow L_{2}(\Omega)} \leq C_{3} c_{*}^{-1 / 2} \varepsilon, \\
\varepsilon^{2}\left\|F(k)^{\perp}\left(S(k, \varepsilon) P+\varepsilon^{2} I\right)^{-1} P\right\|_{L_{2}(\Omega) \rightarrow L_{2}(\Omega)} \leq C_{4} c_{*}^{-1 / 2} \varepsilon .
\end{gathered}
$$


Relations (5.15) and (5.16) show that, for the proof of Theorem 4.1, it suffices to estimate the operator $\varepsilon^{2} J(k, \varepsilon)$ with

$$
J(k, \varepsilon):=\left(A(k, \varepsilon)+\varepsilon^{2} I\right)^{-1} P-F(k)\left(S(k, \varepsilon) P+\varepsilon^{2} I\right)^{-1} P .
$$

Thus, Theorem 4.1 is a consequence of the following proposition.

Proposition 5.3. Suppose that conditions of Theorem 4.1 are satisfied. Let $J(k, \varepsilon)$ be the operator defined by (5.17). Then

$$
\varepsilon^{2}\|J(k, \varepsilon)\|_{L_{2}(\Omega) \rightarrow L_{2}(\Omega)} \leq \widehat{C} \varepsilon, \quad|k| \leq t_{0}, \quad 0<\varepsilon \leq 1 .
$$

The constant $\widehat{C}$ depends only on $c_{0}, c_{1}$, and $c_{2}$.

Proposition 5.3 will be proved in $\S 7$.

\section{$\S 6$. Estimates of COMmutators}

6.1. For the proof of Proposition 5.3, we need to estimate the commutator of the operator $D_{2}$ with the resolvent $\left(A(k, \varepsilon)+\varepsilon^{2} I\right)^{-1}$ and with the spectral projection $F(k)$ of the operator $A_{1}(k)$.

Lemma 6.1. Let $|k| \leq t_{0}, \varepsilon>0$. The operator $\operatorname{clos}\left[\left(A(k, \varepsilon)+\varepsilon^{2} I\right)^{-1}, D_{2}\right]$ is bounded on $L_{2}(\Omega)$, and

$$
\begin{aligned}
& \left\|\left[\left(A(k, \varepsilon)+\varepsilon^{2} I\right)^{-1}, D_{2}\right] P^{\perp}\right\|_{L_{2}(\Omega) \rightarrow L_{2}(\Omega)} \\
& \quad=\left\|P^{\perp}\left[\left(A(k, \varepsilon)+\varepsilon^{2} I\right)^{-1}, D_{2}\right]\right\|_{L_{2}(\Omega) \rightarrow L_{2}(\Omega)} \leq C_{5}\left(t^{2}+\varepsilon^{2}\right)^{-1 / 2} .
\end{aligned}
$$

The constant $C_{5}$ depends on $c_{0}, c_{1}$, and $c_{2}$.

Proof. Under conditions (1.1), (1.3), and (1.4), for the functions $u \in \operatorname{Dom} A(k, \varepsilon)$ we have

$$
u \in \widetilde{H}^{1}(\Omega), \quad D_{2} u \in \widetilde{H}^{1}(\Omega) .
$$

This property expresses a partial smoothness improvement (with respect to $x_{2}$ ) of the solutions of the elliptic equation $-\operatorname{div} g(\mathbf{x}) \nabla u=f(\mathbf{x})$ with periodic boundary conditions and $f \in L_{2}(\Omega)$. This is ensured by the fact that the matrix of coefficients $g(\mathbf{x})$ is periodic and satisfies the Lipschitz condition (with respect to $x_{2}$ ). The corresponding theorem about smoothness improvement can easily be established by analogy with the proof of Theorem 10.1 in Chapter III of the book 9 or Theorem 8.8 in 10 .

Let $u, v \in \operatorname{Dom} A(k, \varepsilon)$. Then $D_{2} u, D_{2} v \in \operatorname{Dom} a(k, \varepsilon)$, and

$$
\begin{aligned}
a(k, \varepsilon) & {\left[D_{2} u, v\right]+\varepsilon^{2}\left(D_{2} u, v\right)_{L_{2}(\Omega)}-a(k, \varepsilon)\left[u, D_{2} v\right]-\varepsilon^{2}\left(u, D_{2} v\right)_{L_{2}(\Omega)} } \\
= & \int_{\Omega}\left(g_{1}(\mathbf{x})\left(\left(D_{1}+k\right) D_{2} u\right) \overline{\left(D_{1}+k\right) v}+\varepsilon^{2} g_{2}(\mathbf{x})\left(D_{2}^{2} u\right) \overline{D_{2} v}\right) d \mathbf{x} \\
& +\varepsilon^{2} \int_{\Omega}\left(\left(D_{2} u\right) \bar{v}-u\left(\overline{D_{2} v}\right)\right) d \mathbf{x} \\
& -\int_{\Omega}\left(g_{1}(\mathbf{x})\left(\left(D_{1}+k\right) u\right) \overline{\left(D_{1}+k\right) D_{2} v}+\varepsilon^{2} g_{2}(\mathbf{x})\left(D_{2} u\right) \overline{D_{2}^{2} v}\right) d \mathbf{x} \\
= & -\int_{\Omega}\left(\left(D_{2} g_{1}\right)(\mathbf{x})\left(\left(D_{1}+k\right) u\right) \overline{\left(D_{1}+k\right) v}+\varepsilon^{2}\left(D_{2} g_{2}\right)(\mathbf{x})\left(D_{2} u\right) \overline{D_{2} v}\right) d \mathbf{x} .
\end{aligned}
$$

We have integrated by parts and used the periodic boundary conditions (in $x_{2}$ ) on $u$ and $v$, and also the periodicity of the coefficients $g_{j}(\mathbf{x})$ with respect to $x_{2}$ (see (1.3)). 
Now we plug the functions $u=\left(A(k, \varepsilon)+\varepsilon^{2} I\right)^{-1} \varphi, v=\left(A(k, \varepsilon)+\varepsilon^{2} I\right)^{-1} \psi$ in the relation obtained. Here $\varphi, \psi \in L_{2}(\Omega)$. Then the left-hand side of (6.2) takes the form

$$
\begin{aligned}
& \left(D_{2}\left(A(k, \varepsilon)+\varepsilon^{2} I\right)^{-1} \varphi, \psi\right)_{L_{2}(\Omega)}-\left(\varphi, D_{2}\left(A(k, \varepsilon)+\varepsilon^{2} I\right)^{-1} \psi\right)_{L_{2}(\Omega)} \\
& \quad=\left(\operatorname{clos}\left[D_{2},\left(A(k, \varepsilon)+\varepsilon^{2} I\right)^{-1}\right] \varphi, \psi\right)_{L_{2}(\Omega)},
\end{aligned}
$$

and the right-hand side looks like this:

$$
\begin{gathered}
-\int_{\Omega}\left(D_{2} g_{1}\right)(\mathbf{x})\left(\left(D_{1}+k\right)\left(A(k, \varepsilon)+\varepsilon^{2} I\right)^{-1} \varphi\right) \overline{\left(\left(D_{1}+k\right)\left(A(k, \varepsilon)+\varepsilon^{2} I\right)^{-1} \psi\right)} d \mathbf{x} \\
-\int_{\Omega} \varepsilon^{2}\left(D_{2} g_{2}\right)(\mathbf{x})\left(D_{2}\left(A(k, \varepsilon)+\varepsilon^{2} I\right)^{-1} \varphi\right) \overline{\left(D_{2}\left(A(k, \varepsilon)+\varepsilon^{2} I\right)^{-1} \psi\right)} d \mathbf{x} .
\end{gathered}
$$

Thus,

$$
\begin{aligned}
\operatorname{clos}[ & \left.D_{2},\left(A(k, \varepsilon)+\varepsilon^{2} I\right)^{-1}\right] \\
= & -\left(\left(D_{1}+k\right)\left(A(k, \varepsilon)+\varepsilon^{2} I\right)^{-1}\right)^{*}\left(\left(D_{2} g_{1}\right)\left(D_{1}+k\right)\left(A(k, \varepsilon)+\varepsilon^{2} I\right)^{-1}\right) \\
& -\left(\varepsilon D_{2}\left(A(k, \varepsilon)+\varepsilon^{2} I\right)^{-1}\right)^{*}\left(\left(D_{2} g_{2}\right) \varepsilon D_{2}\left(A(k, \varepsilon)+\varepsilon^{2} I\right)^{-1}\right) .
\end{aligned}
$$

By (1.4), (5.6), (5.8), and (5.9), the operator on the right is bounded.

From (1.4), (5.6), (5.8), (5.9), and (5.13) it follows that the operator (6.3) multiplied by $P^{\perp}$ from the right satisfies the estimate

$$
\left\|\left[D_{2},\left(A(k, \varepsilon)+\varepsilon^{2} I\right)^{-1}\right] P^{\perp}\right\|_{L_{2}(\Omega) \rightarrow L_{2}(\Omega)} \leq 2 c_{0}^{-1} c_{*}^{-1 / 2} C_{3} c_{2}\left(t^{2}+\varepsilon^{2}\right)^{-1 / 2},
$$

which proves (6.1) with $C_{5}=2 c_{0}^{-1} c_{*}^{-1 / 2} C_{3} c_{2}$.

6.2. For calculating the commutator $\left[D_{2}, F(k)\right]$, we represent the spectral projection $F(k)$ as the integral of the resolvent $\left(A_{1}(k)-z I\right)^{-1}$ over the contour $\Gamma$ that envelopes the real interval $[0, \delta]$ equidistantly with distance $\delta$. We recall that, for $|k| \leq t_{0}$, the interval $(\delta, 3 \delta)$ is free of the spectrum of the operator $A_{1}(k)$; therefore,

$$
\left\|\left(A_{1}(k)-z I\right)^{-1}\right\|_{L_{2}(\Omega) \rightarrow L_{2}(\Omega)} \leq \delta^{-1}, \quad|k| \leq t_{0}, \quad z \in \Gamma .
$$

We have

$$
F(k)=-\frac{1}{2 \pi i} \int_{\Gamma}\left(A_{1}(k)-z I\right)^{-1} d z .
$$

Lemma 6.2. Let $|k| \leq t_{0}, \varepsilon>0$. The operator $\operatorname{clos}\left[D_{2}, F(k)\right]$ is bounded on $L_{2}(\Omega)$, and

$$
\begin{gathered}
\left\|\left[D_{2}, F(k)\right]\right\|_{L_{2}(\Omega) \rightarrow L_{2}(\Omega)} \leq C_{6}, \\
\left\|\left[D_{2}, F(k)\right] F(k)\right\|_{L_{2}(\Omega) \rightarrow L_{2}(\Omega)} \leq C_{7} t,
\end{gathered}
$$

where the constants $C_{6}$ and $C_{7}$ depend on $c_{0}, c_{1}$, and $c_{2}$.

Proof. First, we calculate the commutator $\operatorname{clos}\left[D_{2},\left(A_{1}(k)-z I\right)^{-1}\right]$. Suppose functions $u, v \in \operatorname{Dom} a_{1}(k)$ (see (3.2)) are such that $D_{2} u, D_{2} v \in \operatorname{Dom} a_{1}(k)$ and $u(\mathbf{x}), v(\mathbf{x})$ are equal to zero in some neighborhood of the set $\left\{\mathbf{x} \in \partial \Omega: x_{2}=0\right.$ or $\left.x_{2}=a\right\}$. Then

$$
\begin{aligned}
a_{1}(k) & {\left[D_{2} u, v\right]-z\left(D_{2} u, v\right)_{L_{2}(\Omega)}-a_{1}(k)\left[u, D_{2} v\right]+z\left(u, D_{2} v\right)_{L_{2}(\Omega)} } \\
= & \int_{\Omega}\left(g_{1}(\mathbf{x})\left(\left(D_{1}+k\right) D_{2} u\right) \overline{\left(D_{1}+k\right) v}-g_{1}(\mathbf{x})\left(\left(D_{1}+k\right) u\right) \overline{\left(D_{1}+k\right) D_{2} v}\right) d \mathbf{x} \\
& \quad+z \int_{\Omega}\left(u \overline{D_{2} v}-\left(D_{2} u\right) \bar{v}\right) d \mathbf{x} \\
= & -\int_{\Omega}\left(D_{2} g_{1}\right)(\mathbf{x})\left(D_{1}+k\right) u \overline{\left(D_{1}+k\right) v} d \mathbf{x} .
\end{aligned}
$$


We have integrated by parts and used the fact that $u$ and $v$ vanish near the corresponding part of the boundary. We put $u=\left(A_{1}(k)-z I\right)^{-1} \varphi, v=\left(A_{1}(k)-z I\right)^{-1} \psi$, where $\varphi, \psi \in$ $C_{0}^{\infty}(\Omega)$. By using (1.4), it is easily seen that $u$ and $v$ satisfy the required conditions. From (6.8) it follows that

$$
\begin{gathered}
\left(D_{2}\left(A_{1}(k)-z I\right)^{-1} \varphi, \psi\right)_{L_{2}(\Omega)}-\left(\varphi, D_{2}\left(A_{1}(k)-z I\right)^{-1} \psi\right)_{L_{2}(\Omega)} \\
\quad=-\int_{\Omega}\left(D_{2} g_{1}\right)(\mathbf{x})\left(\left(D_{1}+k\right)\left(A_{1}(k)-z I\right)^{-1} \varphi\right) \overline{\left(\left(D_{1}+k\right)\left(A_{1}(k)-z I\right)^{-1} \psi\right)} d \mathbf{x}, \\
\varphi, \psi \in C_{0}^{\infty}(\Omega) .
\end{gathered}
$$

Since $C_{0}^{\infty}(\Omega)$ is dense in $L_{2}(\Omega)$, we obtain

$$
\begin{aligned}
& \operatorname{clos}\left[D_{2},\left(A_{1}(k)-z I\right)^{-1}\right] \\
& \quad=-\left(\left(D_{1}+k\right)\left(A_{1}(k)-z I\right)^{-1}\right)^{*}\left(\left(D_{2} g_{1}\right)\left(D_{1}+k\right)\left(A_{1}(k)-z I\right)^{-1}\right) .
\end{aligned}
$$

Now (1.4) and (5.10) yield the estimate

$$
\begin{aligned}
& \left\|\left[D_{2},\left(A_{1}(k)-z I\right)^{-1}\right]\right\|_{L_{2}(\Omega) \rightarrow L_{2}(\Omega)} \\
& \quad \leq c_{2}\left\|\left(D_{1}+k\right) A_{1}(k)^{-1 / 2}\right\|^{2}\left\|A_{1}(k)^{1 / 2}\left(A_{1}(k)-z I\right)^{-1}\right\|^{2} \\
& \quad \leq c_{2} c_{0}^{-1}\left\|A_{1}(k)^{1 / 2}\left(A_{1}(k)-z I\right)^{-1}\right\|^{2} .
\end{aligned}
$$

Finally, we use the inequality

$$
\left\|A_{1}(k)^{1 / 2}\left(A_{1}(k)-z I\right)^{-1}\right\| \leq c(\delta), \quad z \in \Gamma, \quad|k| \leq t_{0},
$$

where $c(\delta)$ is the maximum value of the function $f(\lambda)=\lambda^{1 / 2}|\lambda-z|^{-1}$ for $\lambda \in[0, \delta] \cup$ $[3 \delta, \infty)$ and $z \in \Gamma$. Inequalities (6.10) and (6.11) imply

$$
\left\|\left[D_{2},\left(A_{1}(k)-z I\right)^{-1}\right]\right\|_{L_{2}(\Omega) \rightarrow L_{2}(\Omega)} \leq c_{2} c_{0}^{-1} c(\delta)^{2}, \quad z \in \Gamma, \quad|k| \leq t_{0} .
$$

By (6.5) and (6.12), we have

$$
\left\|\left[D_{2}, F(k)\right]\right\|_{L_{2}(\Omega) \rightarrow L_{2}(\Omega)} \leq(2 \delta+2 \pi \delta)(2 \pi)^{-1} c_{2} c_{0}^{-1} c(\delta)^{2}=: C_{6}, \quad|k| \leq t_{0},
$$

which proves (6.6).

Relations (1.4), (5.10), (6.4), and (6.11) show that the commutator (6.9) multiplied by $F(k)$ from the right satisfies the estimate

$$
\begin{aligned}
\|\left[D_{2},\right. & \left.\left(A_{1}(k)-z I\right)^{-1}\right] F(k) \|_{L_{2}(\Omega) \rightarrow L_{2}(\Omega)} \\
\leq & c_{2}\left\|\left(D_{1}+k\right) A_{1}(k)^{-1 / 2}\right\|^{2}\left\|A_{1}(k)^{1 / 2}\left(A_{1}(k)-z I\right)^{-1}\right\| \\
& \times\left\|A_{1}(k)^{1 / 2} F(k)\right\|\left\|\left(A_{1}(k)-z I\right)^{-1}\right\| \\
\leq & c_{2} c_{0}^{-1} c(\delta) \delta^{-1}\left\|A_{1}(k)^{1 / 2} F(k)\right\|, \quad z \in \Gamma, \quad|k| \leq t_{0} .
\end{aligned}
$$

Recalling (3.23) and (3.26), we obtain

$$
\begin{aligned}
& \left\|A_{1}(k)^{1 / 2} F(k) u\right\|_{L_{2}(\Omega)}^{2}=\left(A_{1}(k) F(k) u, u\right)_{L_{2}(\Omega)}=\left(\left(t^{2} S P+t^{3} \Psi(k)\right) u, u\right)_{L_{2}(\Omega)} \\
& \quad \leq\left(t^{2}\|S\|_{L_{2}(\Omega) \rightarrow L_{2}(\Omega)}+t^{3}\|\Psi(k)\|_{L_{2}(\Omega) \rightarrow L_{2}(\Omega)}\|u\|_{L_{2}(\Omega)}^{2}\right. \\
& \quad \leq\left(c_{1}+C_{2} t_{0}\right) t^{2}\|u\|_{L_{2}(\Omega)}^{2}, \quad t \leq t_{0}
\end{aligned}
$$

(we have also used the fact that $\|S\|_{L_{2}(\Omega) \rightarrow L_{2}(\Omega)}=\max \left|g_{1}^{0}\left(x_{2}\right)\right| \leq c_{1}$ by (1.9)). Thus,

$$
\left\|A_{1}(k)^{1 / 2} F(k)\right\|_{L_{2}(\Omega) \rightarrow L_{2}(\Omega)} \leq\left(c_{1}+C_{2} t_{0}\right)^{1 / 2} t, \quad|k| \leq t_{0} .
$$

Combining this with (6.5) and (6.14), we arrive at (6.7) with

$$
C_{7}=(2 \delta+2 \pi \delta)(2 \pi)^{-1} c_{2} c_{0}^{-1} c(\delta) \delta^{-1}\left(c_{1}+C_{2} t_{0}\right)^{1 / 2} .
$$


6.3. In the sequel, we shall need the following statement.

Lemma 6.3. We have

$$
F(k) \widetilde{H}^{1}(\Omega) \subset \widetilde{H}^{1}(\Omega) .
$$

Proof. Let $u \in \widetilde{H}^{1}(\Omega)$. Then, obviously, $u \in \operatorname{Dom} a_{1}(k)=\int_{(0, a)} \oplus \widetilde{H}^{1}(0,1) d x_{2}$. Since $F(k) \operatorname{Dom} a_{1}(k) \subset \operatorname{Dom} a_{1}(k)$, a fortiori, we have $F(k) u \in \operatorname{Dom} a_{1}(k)$. This means that $D_{1}(F(k) u) \in L_{2}(\Omega)$, and $F(k) u$ satisfies periodic boundary conditions with respect to $x_{1}$. Next,

$$
D_{2}(F(k) u)=F(k) D_{2} u+\left[D_{2}, F(k)\right] u \in L_{2}(\Omega)
$$

because $D_{2} u \in L_{2}(\Omega)$, and the operators $F(k)$ and $\left[D_{2}, F(k)\right]$ are bounded in $L_{2}(\Omega)$. The function $F(k) u$ satisfies periodic boundary conditions in $x_{2}$ because $u(\mathbf{x})$ is periodic in $x_{2}$, the operator $F(k)$ acts in the direct integral (3.3) layerwise, and the coefficient $g_{1}(\mathbf{x})$ is periodic with respect to $x_{2}$ (see (1.3)).

\section{§7. Proof of Proposition 5.3}

7.1. For the study of the operator (5.17), we wish to apply the identity

$$
\begin{aligned}
J(k, \varepsilon)= & \left(A(k, \varepsilon)+\varepsilon^{2} I\right)^{-1}\left(S(k, \varepsilon) P+\varepsilon^{2} P-\left(A(k, \varepsilon)+\varepsilon^{2} I\right) F(k)\right) \\
& \times\left(S(k, \varepsilon) P+\varepsilon^{2} I\right)^{-1} P .
\end{aligned}
$$

However, strictly speaking, the operator in the central brackets makes no sense. In order to obtain a precise version of (7.1), we need the following statement of abstract nature.

Lemma 7.1. Let $\mathfrak{t}_{1}$ and $\mathfrak{t}_{2}$ be two closed and densely defined sesquilinear forms in a separable Hilbert space $\mathfrak{H}$. Suppose that both quadratic forms $\mathfrak{t}_{1}[u, u]$ and $\mathfrak{t}_{2}[u, u]$ are positive definite. Let $T_{1}$ and $T_{2}$ denote the selfadjoint operators in $\mathfrak{H}$ corresponding to the forms $\mathfrak{t}_{1}$ and $\mathfrak{t}_{2}$, respectively, and let $\mathcal{P}$ and $\mathcal{Q}$ be orthoprojections in $\mathfrak{H}$. Assume the following conditions:

$1^{\circ} \mathfrak{d}:=\operatorname{Dom}_{1} \subset \operatorname{Dom}_{2}$

$2^{\circ} \mathcal{P}$ Dom $_{2} \subset$ Dom $_{1}$

$3^{\circ} \mathcal{Q} \mathfrak{d} \subset \mathfrak{d}$

$4^{\circ} \mathcal{P}$ commutes with $T_{2}$;

$5^{\circ}$ the following representation is true:

$$
\mathfrak{t}_{2}[\mathcal{P} u, v]-\mathfrak{t}_{1}[\mathcal{Q} u, v]=\left(G_{0} u, G v\right)_{\mathfrak{G}}, \quad u, v \in \mathfrak{d},
$$

where $G_{0}, G: \mathfrak{H} \rightarrow \mathfrak{G}$ are densely defined linear operators acting from $\mathfrak{H}$ into some separable Hilbert space $\mathfrak{G}$. Moreover, $\mathfrak{d} \subset \operatorname{Dom} G_{0}, \mathfrak{d} \subset \operatorname{Dom} G$, and the operators

$$
G T_{1}^{-1}: \mathfrak{H} \rightarrow \mathfrak{G}, \quad G_{0} T_{2}^{-1} \mathcal{P}: \mathfrak{H} \rightarrow \mathfrak{G}
$$

are bounded.

Then we have

$$
T_{1}^{-1} \mathcal{P}-\mathcal{Q} T_{2}^{-1} \mathcal{P}=\left(G T_{1}^{-1}\right)^{*}\left(G_{0} T_{2}^{-1} \mathcal{P}\right)
$$

Proof. In (7.2) we take $u=\mathcal{P} T_{2}^{-1} \varphi$ and $v=T_{1}^{-1} \psi$, where $\varphi, \psi \in \mathfrak{H}$. Then $u \in \mathfrak{d}$ by condition $2^{\circ}$, and obviously $v \in \mathfrak{d}$. Thus,

$$
\mathfrak{t}_{2}\left[\mathcal{P} T_{2}^{-1} \varphi, T_{1}^{-1} \psi\right]-\mathfrak{t}_{1}\left[\mathcal{Q P} T_{2}^{-1} \varphi, T_{1}^{-1} \psi\right]=\left(G_{0} \mathcal{P} T_{2}^{-1} \varphi, G T_{1}^{-1} \psi\right)_{\mathfrak{G}}, \quad \varphi, \psi \in \mathfrak{H} .
$$

Using condition $4^{\circ}$, we rewrite this as

$$
\left(\mathcal{P} \varphi, T_{1}^{-1} \psi\right)_{\mathfrak{H}}-\left(\mathcal{Q} T_{2}^{-1} \mathcal{P} \varphi, \psi\right)_{\mathfrak{H}}=\left(G_{0} T_{2}^{-1} \mathcal{P} \varphi, G T_{1}^{-1} \psi\right)_{\mathfrak{G}}, \quad \varphi, \psi \in \mathfrak{H},
$$

which implies (7.3). 
7.2. Let $|k| \leq t_{0}, \varepsilon>0$. We are going to apply Lemma 7.1 with $\mathfrak{H}=L_{2}(\Omega), \mathcal{P}=P$, $\mathcal{Q}=F(k)$,

$$
\begin{aligned}
& \mathfrak{t}_{1}[u, v]=a(k, \varepsilon)[u, v]+\varepsilon^{2}(u, v)_{L_{2}(\Omega)}, \\
& \mathfrak{t}_{2}[u, v]=s(k, \varepsilon)[P u, P v]+\varepsilon^{2}(u, v)_{L_{2}(\Omega)},
\end{aligned}
$$

$\mathfrak{d}=\widetilde{H}^{1}(\Omega)$, and Dom $\mathfrak{t}_{2}=\left\{u \in L_{2}(\Omega): P u \in \widetilde{H}^{1}(0, a)\right\}$. Obviously, conditions $1^{\circ}, 2^{\circ}$, and $4^{\circ}$ are satisfied. Condition $3^{\circ}$ is also satisfied by Lemma 6.3. We check condition $5^{\circ}$. The left-hand side of (7.2) takes the form

$$
\begin{aligned}
\mathfrak{A}[u, v] & :=s(k, \varepsilon)[P u, P v]+\varepsilon^{2}(P u, v)_{L_{2}(\Omega)}-a(k, \varepsilon)[F(k) u, v]-\varepsilon^{2}(F(k) u, v)_{L_{2}(\Omega)} \\
& =\mathcal{I}_{1}[u, v]+\mathcal{I}_{2}[u, v]+\mathcal{I}_{3}[u, v]
\end{aligned}
$$

where, in accordance with (2.2), (3.1), and (4.1),

$$
\begin{gathered}
\mathcal{I}_{1}[u, v]:=\varepsilon^{2}((P-F(k)) u, v)_{L_{2}(\Omega)}, \\
\mathcal{I}_{2}[u, v]:=t^{2}(S P u, P v)_{L_{2}(\Omega)}-a_{1}(k)[F(k) u, v], \\
\mathcal{I}_{3}[u, v]:=\left(g_{2}^{0} \varepsilon D_{2} P u, \varepsilon D_{2} P v\right)_{L_{2}(\Omega)}-\left(g_{2} \varepsilon D_{2} F(k) u, \varepsilon D_{2} v\right)_{L_{2}(\Omega)} .
\end{gathered}
$$

The forms $\mathcal{I}_{1}$ and $\mathcal{I}_{2}$. By (3.21), for the form (7.5) we have

$$
\mathcal{I}_{1}[u, v]=-\varepsilon^{2} t(\Phi(k) u, v)_{L_{2}(\Omega)}=\left(G_{01} u, G_{1} v\right)_{L_{2}(\Omega)},
$$

where $G_{01}=-\varepsilon t^{1 / 2} \Phi(k), G_{1}=\varepsilon t^{1 / 2} I$. Using (3.25), (5.6), and (5.7), we obtain

$$
\begin{gathered}
\varepsilon\left\|G_{01}\left(S(k, \varepsilon) P+\varepsilon^{2} I\right)^{-1} P\right\|_{L_{2}(\Omega) \rightarrow L_{2}(\Omega)} \leq C_{1} \varepsilon^{2} t^{1 / 2} c_{*}^{-1}\left(t^{2}+\varepsilon^{2}\right)^{-1} \leq C_{1} c_{*}^{-1} \varepsilon^{1 / 2}, \\
\varepsilon\left\|G_{1}\left(A(k, \varepsilon)+\varepsilon^{2} I\right)^{-1} P\right\|_{L_{2}(\Omega) \rightarrow L_{2}(\Omega)} \leq \varepsilon^{2} t^{1 / 2} c_{*}^{-1}\left(t^{2}+\varepsilon^{2}\right)^{-1} \leq c_{*}^{-1} \varepsilon^{1 / 2} .
\end{gathered}
$$

As to the form (7.6), we apply (3.23):

$$
\mathcal{I}_{2}[u, v]=-t^{3}(\Psi(k) u, v)_{L_{2}(\Omega)}=\left(G_{02} u, G_{2} v\right)_{L_{2}(\Omega)},
$$

where $G_{02}=-t^{3 / 2} \Psi(k), G_{2}=t^{3 / 2} I$. Then, by (3.26) and (5.7),

$$
\begin{aligned}
& \varepsilon\left\|G_{02}\left(S(k, \varepsilon) P+\varepsilon^{2} I\right)^{-1} P\right\|_{L_{2}(\Omega) \rightarrow L_{2}(\Omega)} \\
& \quad \leq C_{2} \varepsilon t^{3 / 2} c_{*}^{-1}\left(t^{2}+\varepsilon^{2}\right)^{-1} \leq C_{2} c_{*}^{-1} \varepsilon^{1 / 2} .
\end{aligned}
$$

From (5.6) we deduce the estimate

$$
\varepsilon\left\|G_{2}\left(A(k, \varepsilon)+\varepsilon^{2} I\right)^{-1} P\right\|_{L_{2}(\Omega) \rightarrow L_{2}(\Omega)} \leq \varepsilon t^{3 / 2} c_{*}^{-1}\left(t^{2}+\varepsilon^{2}\right)^{-1} \leq c_{*}^{-1} \varepsilon^{1 / 2} .
$$

The form $\mathcal{I}_{3}$. We rewrite the form (7.7) as

$$
\mathcal{I}_{3}[u, v]=\mathcal{I}_{3}^{\prime}[u, v]+\mathcal{I}_{4}[u, v],
$$

where

$$
\begin{gathered}
\mathcal{I}_{3}^{\prime}[u, v]=-\left(g_{2} \varepsilon D_{2}(F(k)-P) u, \varepsilon D_{2} v\right)_{L_{2}(\Omega)}, \\
\mathcal{I}_{4}[u, v]=\left(g_{2}^{0} \varepsilon D_{2} P u, \varepsilon D_{2} P v\right)_{L_{2}(\Omega)}-\left(g_{2} \varepsilon D_{2} P u, \varepsilon D_{2} v\right)_{L_{2}(\Omega)} .
\end{gathered}
$$

For the form (7.15), we have

$$
\mathcal{I}_{3}^{\prime}[u, v]=\left(G_{03} u, G_{3} v\right)_{L_{2}(\Omega)},
$$

where $G_{03}=-g_{2} \varepsilon^{1 / 2} D_{2}(F(k)-P), G_{3}=\varepsilon^{3 / 2} D_{2}$. By (3.21), we can represent the operator $G_{03}$ as

$$
G_{03}=\widetilde{G}_{03}+\widehat{G}_{03}+\check{G}_{03},
$$


where

$$
\begin{aligned}
& \widetilde{G}_{03}=-g_{2} \varepsilon^{1 / 2}\left[D_{2}, F(k)\right] F(k), \\
& \widehat{G}_{03}=-g_{2} \varepsilon^{1 / 2}\left[D_{2}, F(k)\right] F(k)^{\perp}, \\
& \check{G}_{03}=-g_{2} \varepsilon^{1 / 2}(F(k)-P) D_{2}=-g_{2} \varepsilon^{1 / 2} t \Phi(k) D_{2} .
\end{aligned}
$$

From (1.1), (5.7), and (6.7) it follows that

$$
\varepsilon\left\|\widetilde{G}_{03}\left(S(k, \varepsilon) P+\varepsilon^{2} I\right)^{-1} P\right\|_{L_{2}(\Omega) \rightarrow L_{2}(\Omega)} \leq \varepsilon^{3 / 2} c_{1} C_{7} t c_{*}^{-1}\left(t^{2}+\varepsilon^{2}\right)^{-1} \leq c_{1} C_{7} c_{*}^{-1} \varepsilon^{1 / 2} .
$$

Relations (1.1), (5.16), and (6.6) yield the estimate

$$
\varepsilon\left\|\widehat{G}_{03}\left(S(k, \varepsilon) P+\varepsilon^{2} I\right)^{-1} P\right\|_{L_{2}(\Omega) \rightarrow L_{2}(\Omega)} \leq c_{1} C_{6} C_{4} c_{*}^{-1 / 2} \varepsilon^{1 / 2} .
$$

Finally, relations (1.1), (3.25), (5.7), and (5.11) imply

$$
\begin{aligned}
& \varepsilon\left\|\check{G}_{03}\left(S(k, \varepsilon) P+\varepsilon^{2} I\right)^{-1} P\right\|_{L_{2}(\Omega) \rightarrow L_{2}(\Omega)} \\
& \quad \leq \varepsilon^{1 / 2} c_{1} C_{1} t c_{0}^{-1 / 2} c_{*}^{-1 / 2}\left(t^{2}+\varepsilon^{2}\right)^{-1 / 2} \leq c_{1} C_{1} c_{0}^{-1 / 2} c_{*}^{-1 / 2} \varepsilon^{1 / 2} .
\end{aligned}
$$

As a result, from (7.18)-(7.21) we obtain

$$
\begin{gathered}
\varepsilon\left\|G_{03}\left(S(k, \varepsilon) P+\varepsilon^{2} I\right)^{-1} P\right\|_{L_{2}(\Omega) \rightarrow L_{2}(\Omega)} \leq C_{8} \varepsilon^{1 / 2}, \\
C_{8}=c_{1} c_{*}^{-1 / 2}\left(C_{7} c_{*}^{-1 / 2}+C_{4} C_{6}+C_{1} c_{0}^{-1 / 2}\right) .
\end{gathered}
$$

Next, by (5.6) and (5.9),

$$
\begin{aligned}
\varepsilon\left\|G_{3}\left(A(k, \varepsilon)+\varepsilon^{2} I\right)^{-1}\right\|_{L_{2}(\Omega) \rightarrow L_{2}(\Omega)} & =\varepsilon^{3 / 2}\left\|\varepsilon D_{2}\left(A(k, \varepsilon)+\varepsilon^{2} I\right)^{-1}\right\|_{L_{2}(\Omega) \rightarrow L_{2}(\Omega)} \\
\leq \varepsilon^{3 / 2} c_{0}^{-1 / 2} c_{*}^{-1 / 2}\left(t^{2}+\varepsilon^{2}\right)^{-1 / 2} & \leq c_{0}^{-1 / 2} c_{*}^{-1 / 2} \varepsilon^{1 / 2} .
\end{aligned}
$$

The form (7.16) can be rewritten as

$$
\begin{aligned}
\mathcal{I}_{4}[u, v]= & \left(g_{2}^{0} \varepsilon D_{2} P u, \varepsilon D_{2} P v\right)_{L_{2}(\Omega)} \\
& -\left(g_{2} \varepsilon D_{2} P u, P \varepsilon D_{2} v\right)_{L_{2}(\Omega)}-\left(g_{2} \varepsilon D_{2} P u, P^{\perp} \varepsilon D_{2} v\right)_{L_{2}(\Omega)} .
\end{aligned}
$$

Since $P g_{2} P=g_{2}^{0}$ (see (1.8)), the first two terms on the right in (7.24) compensate each other. Consequently,

$$
\mathcal{I}_{4}[u, v]=-\left(g_{2} \varepsilon D_{2} P u, P^{\perp} \varepsilon D_{2} v\right)_{L_{2}(\Omega)}=\left(G_{04} u, G_{4} v\right)_{L_{2}(\Omega)},
$$

where $G_{04}=-g_{2} \varepsilon^{3 / 2} D_{2} P, G_{4}=\varepsilon^{1 / 2} P^{\perp} D_{2}$. Relations (1.1), (5.7), and (5.11) imply that

$$
\begin{aligned}
& \varepsilon\left\|G_{04}\left(S(k, \varepsilon) P+\varepsilon^{2} I\right)^{-1} P\right\|_{L_{2}(\Omega) \rightarrow L_{2}(\Omega)} \\
& \quad \leq \varepsilon^{3 / 2} c_{1} c_{0}^{-1 / 2} c_{*}^{-1 / 2}\left(t^{2}+\varepsilon^{2}\right)^{-1 / 2} \leq c_{1} c_{0}^{-1 / 2} c_{*}^{-1 / 2} \varepsilon^{1 / 2} .
\end{aligned}
$$

Next, we have

$$
G_{4}\left(A(k, \varepsilon)+\varepsilon^{2} I\right)^{-1}=\varepsilon^{1 / 2} P^{\perp}\left[D_{2},\left(A(k, \varepsilon)+\varepsilon^{2} I\right)^{-1}\right]+\varepsilon^{1 / 2} P^{\perp}\left(A(k, \varepsilon)+\varepsilon^{2} I\right)^{-1} D_{2} .
$$

The first term on the right is estimated with the help of (6.1), and the second with the help of (5.13) and (5.9). As a result, we obtain

$$
\begin{aligned}
& \varepsilon\left\|G_{4}\left(A(k, \varepsilon)+\varepsilon^{2} I\right)^{-1}\right\|_{L_{2}(\Omega) \rightarrow L_{2}(\Omega)} \\
& \quad \leq \varepsilon^{3 / 2} C_{5}\left(t^{2}+\varepsilon^{2}\right)^{-1 / 2}+\varepsilon^{1 / 2} c_{0}^{-1 / 2} C_{3} \leq\left(C_{5}+c_{0}^{-1 / 2} C_{3}\right) \varepsilon^{1 / 2} .
\end{aligned}
$$


7.3. We summarize the study of the form (7.4). By (7.8), (7.11), (7.14), (7.17), and (7.25), this form can be represented as

$$
\begin{aligned}
\mathfrak{A}[u, v]= & \left(G_{01} u, G_{1} v\right)_{L_{2}(\Omega)} \\
& +\left(G_{02} u, G_{2} v\right)_{L_{2}(\Omega)}+\left(G_{03} u, G_{3} v\right)_{L_{2}(\Omega)}+\left(G_{04} u, G_{4} v\right)_{L_{2}(\Omega)} .
\end{aligned}
$$

We put $\mathfrak{G}=L_{2}\left(\Omega ; \mathbb{C}^{4}\right)=L_{2}(\Omega) \times L_{2}(\Omega) \times L_{2}(\Omega) \times L_{2}(\Omega)$ and introduce the operators

$$
\begin{gathered}
G_{0}: L_{2}(\Omega) \rightarrow \mathfrak{G}, \quad G_{0}=\operatorname{col}\left\{G_{01}, G_{02}, G_{03}, G_{04}\right\}, \\
G: L_{2}(\Omega) \rightarrow \mathfrak{G}, \quad G=\operatorname{col}\left\{G_{1}, G_{2}, G_{3}, G_{4}\right\}, \\
\operatorname{Dom} G_{0}=\operatorname{Dom} G=\left\{u \in L_{2}(\Omega): D_{2} u \in L_{2}(\Omega)\right\} .
\end{gathered}
$$

The operators $G_{0}$ and $G$ are densely defined, and we have $\mathfrak{d}=\widetilde{H}^{1}(\Omega) \subset \operatorname{Dom} G_{0}=$ Dom $G$ and

$$
\mathfrak{A}[u, v]=\left(G_{0} u, G v\right)_{\mathfrak{G}}, \quad u, v \in \mathfrak{d} .
$$

Thus, we have checked condition (7.2) in the case in question. Relations (7.10), (7.13), (7.23), and (7.27) show that the operator $G\left(A(k, \varepsilon)+\varepsilon^{2} I\right)^{-1}: L_{2}(\Omega) \rightarrow \mathfrak{G}$ is bounded, and

$$
\varepsilon\left\|G\left(A(k, \varepsilon)+\varepsilon^{2} I\right)^{-1}\right\|_{L_{2}(\Omega) \rightarrow \mathfrak{G}} \leq C_{9} \varepsilon^{1 / 2},
$$

where $C_{9}^{2}=2 c_{*}^{-2}+c_{0}^{-1} c_{*}^{-1}+\left(C_{5}+c_{0}^{-1 / 2} C_{3}\right)^{2}$. Inequalities (7.9), (7.12), (7.22), and (7.26) imply that the operator $G_{0}\left(S(k, \varepsilon) P+\varepsilon^{2} I\right)^{-1} P: L_{2}(\Omega) \rightarrow \mathfrak{G}$ is bounded, and

$$
\varepsilon\left\|G_{0}\left(S(k, \varepsilon) P+\varepsilon^{2} I\right)^{-1} P\right\|_{L_{2}(\Omega) \rightarrow \mathfrak{G}} \leq C_{10} \varepsilon^{1 / 2},
$$

where $C_{10}^{2}=\left(C_{1}^{2}+C_{2}^{2}\right) c_{*}^{-2}+C_{8}^{2}+c_{1}^{2} c_{0}^{-1} c_{*}^{-1}$. Observe that the constants $C_{9}$ and $C_{10}$ can be expressed in terms of $c_{0}, c_{1}, c_{2}$.

Thus, we see that condition $5^{\circ}$ of Lemma 7.1 is satisfied. Applying Lemma 7.1, we obtain the representation

$$
\begin{aligned}
& \left(A(k, \varepsilon)+\varepsilon^{2} I\right)^{-1} P-F(k)\left(S(k, \varepsilon) P+\varepsilon^{2} I\right)^{-1} P \\
& \quad=\left(G\left(A(k, \varepsilon)+\varepsilon^{2} I\right)^{-1}\right)^{*}\left(G_{0}\left(S(k, \varepsilon) P+\varepsilon^{2} I\right)^{-1} P\right) .
\end{aligned}
$$

Relations (7.28)-(7.30) imply estimate (5.18) with $\widehat{C}=C_{9} C_{10}$. This completes the proof of Proposition 5.3 and, with it, of Theorem 4.1. Also, this completes (see Subsection 4.2) the proof of Theorem 2.1, and, thereby (see Subsection 2.3), that of Theorem 1.1.

\section{§8. On Generalizations of Theorem 1.1}

In this section, we briefly discuss possible generalizations of the main result of the paper.

8.1. The two-dimensional operator of the form $(0.2)$ in $L_{2}(\Pi)$ with a nondiagonal matrix $g(\mathbf{x})$ can also be studied. The statement of Theorem 1.1 carries over to the case where the antidiagonal entries of the matrix $g(\mathbf{x})$ do not depend on $x_{1}$, i.e., $g(\mathbf{x})$ is of the form

$$
g(\mathbf{x})=\left(\begin{array}{cc}
g_{1}\left(x_{1}, x_{2}\right) & \widehat{g}\left(x_{2}\right) \\
\widehat{g}\left(x_{2}\right) & g_{2}\left(x_{1}, x_{2}\right)
\end{array}\right) .
$$

We suppose that the matrix $g(\mathbf{x})$ is positive definite and bounded, and that the coefficients $g_{1}, g_{2}$ are subject to the same conditions as before. Also, we assume that the coefficient $\widehat{g}\left(x_{2}\right)$ is real-valued, periodic (with respect to $x_{2}$ ), and Lipschitz. In this case the effective operator coincides with $A^{0}=-\operatorname{div} g^{0} \operatorname{grad}$, where

$$
g^{0}=\left(\begin{array}{cc}
g_{1}^{0}\left(x_{2}\right) & \widehat{g}\left(x_{2}\right) \\
\widehat{g}\left(x_{2}\right) & g_{2}^{0}\left(x_{2}\right)
\end{array}\right),
$$


and the $g_{j}^{0}\left(x_{2}\right), j=1,2$, are as in (1.7) and (1.8). Under the above conditions, an analog of estimate (1.11) is valid.

In the case where $g(\mathbf{x})$ is a nondiagonal matrix of general type, additional technical difficulties arise, and the author has not yet succeeded in overcoming them.

8.2. An analog of the operator (0.2) can also be studied in the domain $\Xi=\mathbb{R}^{d_{1}} \times(0, a)^{d_{2}}$ of an arbitrary dimension $d=d_{1}+d_{2}$. We use the notation $\mathbf{x}=\left(\mathbf{x}^{\prime}, \mathbf{x}^{\prime \prime}\right) \in \Xi, \mathbf{x}^{\prime} \in \mathbb{R}^{d_{1}}$, $\mathbf{x}^{\prime \prime} \in(0, a)^{d_{2}}, \mathbf{D}_{\mathbf{x}^{\prime}}=-i \operatorname{grad}_{\mathbf{x}^{\prime}}, \mathbf{D}_{\mathbf{x}^{\prime}}^{*}=-i \operatorname{div}_{\mathbf{x}^{\prime}}$, and similarly for $\mathbf{D}_{\mathbf{x}^{\prime \prime}}, \mathbf{D}_{\mathbf{x}^{\prime \prime}}^{*}$. In $L_{2}(\Xi)$, we consider the operator $A$ corresponding to the differential expression

$$
A=\mathbf{D}_{\mathbf{x}^{\prime}}^{*} g_{1}\left(\mathbf{x}^{\prime}, \mathbf{x}^{\prime \prime}\right) \mathbf{D}_{\mathbf{x}^{\prime}}+\mathbf{D}_{\mathbf{x}^{\prime \prime}}^{*} g_{2}\left(\mathbf{x}^{\prime}, \mathbf{x}^{\prime \prime}\right) \mathbf{D}_{\mathbf{x}^{\prime \prime}},
$$

with periodic boundary conditions on $\partial \Xi$. Here $g_{1}(\mathbf{x})$ is a $\left(d_{1} \times d_{1}\right)$-matrix-valued function, and $g_{2}(\mathbf{x})$ is a $\left(d_{2} \times d_{2}\right)$-matrix-valued function in $\Xi$. Both matrices are real-valued, measurable, positive definite, and periodic in $\mathbf{x}^{\prime}$ with respect to a lattice $\Gamma \subset \mathbb{R}^{d_{1}}$. We assume that

$$
g_{j}, g_{j}^{-1} \in L_{\infty}(\Xi), \quad j=1,2 .
$$

Moreover, we assume that $g_{1}, g_{2}$ belong to the Lipschitz class with respect to $\mathbf{x}^{\prime \prime}$,

$$
\underset{\mathbf{x} \in \bar{\Xi}}{\operatorname{ess} \sup }\left|\mathbf{D}_{\mathbf{x}^{\prime \prime}} g_{j}(\mathbf{x})\right| \leq c, \quad j=1,2
$$

and satisfy periodic boundary conditions in $\mathbf{x}^{\prime \prime}$ (the values of $g_{1}, g_{2}$ on the opposite faces of $\Xi$ coincide). We consider the operator

$$
A_{\varepsilon}=\mathbf{D}_{\mathbf{x}^{\prime}}^{*} g_{1}\left(\mathbf{x}^{\prime} / \varepsilon, \mathbf{x}^{\prime \prime}\right) \mathbf{D}_{\mathbf{x}^{\prime}}+\mathbf{D}_{\mathbf{x}^{\prime \prime}}^{*} g_{2}\left(\mathbf{x}^{\prime} / \varepsilon, \mathbf{x}^{\prime \prime}\right) \mathbf{D}_{\mathbf{x}^{\prime \prime}}, \quad \varepsilon>0 .
$$

Theorem 1.1 can be carried over to the operator (8.2). The effective operator has the form

$$
A^{0}=\mathbf{D}_{\mathbf{x}^{\prime}}^{*} g_{1}^{0}\left(\mathbf{x}^{\prime \prime}\right) \mathbf{D}_{\mathbf{x}^{\prime}}+\mathbf{D}_{\mathbf{x}^{\prime \prime}}^{*} g_{2}^{0}\left(\mathbf{x}^{\prime \prime}\right) \mathbf{D}_{\mathbf{x}^{\prime \prime}} .
$$

We present the rule of calculation of the coefficients. Let $\Omega^{\prime} \subset \mathbb{R}^{d_{1}}$ be the cell of the lattice $\Gamma$. Then $g_{2}^{0}\left(\mathbf{x}^{\prime \prime}\right)$ is equal to the mean value of $g_{2}$ :

$$
g_{2}^{0}\left(\mathbf{x}^{\prime \prime}\right)=\left|\Omega^{\prime}\right|^{-1} \int_{\Omega^{\prime}} g_{2}\left(\mathbf{x}^{\prime}, \mathbf{x}^{\prime \prime}\right) d \mathbf{x}^{\prime} .
$$

The matrix $g_{1}(\mathbf{x})$ is homogenized by the rule corresponding to the $d_{1}$-dimensional elliptic operator $\mathbf{D}_{\mathbf{x}^{\prime}}^{*} g_{1} \mathbf{D}_{\mathbf{x}^{\prime}}$. This rule can be described as follows. For a given $\mathbf{C} \in \mathbb{C}^{d_{1}}$, let $v(\mathbf{x})$ be the periodic (in $\mathbf{x}^{\prime}$ ) solution of the equation $\mathbf{D}_{\mathbf{x}^{\prime}}^{*} g_{1}\left(\mathbf{x}^{\prime}, \mathbf{x}^{\prime \prime}\right)\left(\mathbf{D}_{\mathbf{x}^{\prime}} v+\mathbf{C}\right)=0$. (Here $\mathbf{x}^{\prime \prime}$ is a parameter.) Then

$$
g_{1}^{0}\left(\mathbf{x}^{\prime \prime}\right) \mathbf{C}=\left|\Omega^{\prime}\right|^{-1} \int_{\Omega^{\prime}} g_{1}\left(\mathbf{x}^{\prime}, \mathbf{x}^{\prime \prime}\right)\left(\mathbf{D}_{\mathbf{x}^{\prime}} v+\mathbf{C}\right) d \mathbf{x}^{\prime}, \quad \mathbf{C} \in \mathbb{C}^{d_{1}} .
$$

Note that, for $d_{1}=1$, the rule (8.4) corresponds to calculating the harmonic average value of $g_{1}$, and then (8.4) turns into (1.7). The following analog of Theorem 1.1 is true.

Theorem 8.1. Under the above conditions, we have

$$
\left\|\left(A_{\varepsilon}+I\right)^{-1}-\left(A^{0}+I\right)^{-1}\right\|_{L_{2}(\Xi) \rightarrow L_{2}(\Xi)} \leq C \varepsilon, \quad 0<\varepsilon \leq 1 .
$$


8.3. We can include "antidiagonal" terms in the operator (8.1), but only with coefficients independent of $\mathbf{x}^{\prime}$. In this case, $A=-\operatorname{div} g(\mathbf{x}) \operatorname{grad}$, and the matrix $g(\mathbf{x})$ is of the form

$$
g(\mathbf{x})=\left(\begin{array}{cc}
g_{1}\left(\mathbf{x}^{\prime}, \mathbf{x}^{\prime \prime}\right) & \widehat{g}\left(\mathbf{x}^{\prime \prime}\right) \\
\left(\widehat{g}\left(\mathbf{x}^{\prime \prime}\right)\right)^{*} & g_{2}\left(\mathbf{x}^{\prime}, \mathbf{x}^{\prime \prime}\right)
\end{array}\right)
$$

where $g_{1}, g_{2}$ are as above, the $(d \times d)$-matrix-valued function $g(\mathbf{x})$ is bounded and positive definite, and the $\left(d_{1} \times d_{2}\right)$-matrix-valued function $\widehat{g}\left(\mathbf{x}^{\prime \prime}\right)$ is periodic (in $\left.\mathbf{x}^{\prime \prime}\right)$ and belongs to the Lipschitz class. In this case, the effective operator is of the form $A^{0}=-\operatorname{div} g^{0}\left(\mathbf{x}^{\prime \prime}\right) \operatorname{grad}$, where

$$
g^{0}\left(\mathbf{x}^{\prime \prime}\right)=\left(\begin{array}{cc}
g_{1}^{0}\left(\mathbf{x}^{\prime \prime}\right) & \widehat{g}\left(\mathbf{x}^{\prime \prime}\right) \\
\left(\widehat{g}\left(\mathbf{x}^{\prime \prime}\right)\right)^{*} & g_{2}^{0}\left(\mathbf{x}^{\prime \prime}\right)
\end{array}\right)
$$

Here $g_{1}^{0}$ and $g_{2}^{0}$ are defined by the same rules (8.4) and (8.3), respectively. Under the above conditions, an analog of estimate (8.5) is valid.

\section{REFERENCES}

[1] A. Bensoussan, J. L. Lions, and G. Papanicolaou, Asymptotic analysis for periodic structures, Stud. Math. Appl., vol. 5, North-Holland Publishing Co., Amsterdam-New York, 1978. MR0503330 (82h:35001)

[2] N. S. Bakhvalov and G. P. Panasenko, Homogenization: averaging processes in periodic media. Mathematical problems in mechanics of composite materials, "Nauka," Moscow, 1984; English transl., Math. Appl. (Soviet Ser.), vol. 36, Kluwer Acad. Publ. Group, Dordrecht, 1989. MR1112788 (92d:73002)

[3] V. V. Zhikov, S. M. Kozlov, and O. A. Oleŭnik, Homogenization of differential operators, Fizmatgiz, Moscow, 1993; English transl., Springer-Verlag, Berlin, 1994. MR1329546 (96h:35003b)

[4] V. V. Zhikov, Spectral approach to asymptotic diffusion problems, Differentsial'nye Uravneniya 25 (1989), no. 1, 44-50; English transl., Differential Equations 25 (1989), no. 1, 33-39. MR0986395 (90a:35107)

[5] C. Conca and M. Vanninathan, Homogenization of periodic structures via Bloch decomposition, SIAM J. Appl. Math. 57 (1997), no. 6, 1639-1659. MR1484944 (98j:35017)

[6] E. V. Sevost'yanova, Asymptotic expansion of the solution of a second-order elliptic equation with periodic rapidly oscillating coefficients, Mat. Sb. (N.S.) 115 (1981), no. 2, 204-222; English transl., Math. USSR-Sb. 43 (1982), no. 2, 181-198. MR.0622145 (83d:35038)

[7] M. Birman and T. Suslina, Threshold effects near the lower edge of the spectrum for periodic differential operators of mathematical physics, Systems, Approximation, Singular Integral Operators, and Related Topics (Bordeaux, 2000), Oper. Theory Adv. Appl., vol. 129, Birkhäuser, Basel, 2001, pp. 71-107. MR 1882692 (2003f:35220)

[8] - Second order periodic differential operators. Threshold properties and homogenization, Algebra i Analiz 15 (2003), no. 5, 1-108; English transl., St. Petersburg Math. J. 15 (2004), no. 5, 1-77. MR,2068790

[9] O. A. Ladyzhenskaya and N. N. Ural'tseva, Linear and quasilinear equations of elliptic type, 2nd ed., "Nauka", Moscow, 1973; English transl. of 1st ed., Acad. Press, New York-London, 1968. MR.0509265 (58:23009)

[10] D. Gilbarg and N. S. Trudinger, Elliptic partial differential equations of second order, SpringerVerlag, Berlin, 1998. MR0737190 (86c:35035)

St. Petersburg State University, Faculty of Physics, Petrodvorets, Ul'yanovskaya 1, St. Petersburg 198504, Russia

E-mail address: tanya@petrov.stoic.spb.su

Received 1/SEP/2003

Translated by THE AUTHOR 